\title{
Abelian decomposition and Weyl symmetric effective action of $\mathrm{SU}(3) \mathrm{QCD}$
}

\author{
Y. M. Cho ${ }^{1,2, a} \mathbb{C}_{0}$, Franklin H. Cho ${ }^{3}$ \\ ${ }^{1}$ Center for Quantum Spacetime, Sogang University, Seoul 04107, Korea \\ 2 School of Physics and Astronomy, Seoul National University, Seoul 08826, Korea \\ ${ }^{3}$ Department of Physics and Astronomy, Institute for Quantum Computing, University of Waterloo, Waterloo, ON N2L 3G1, Canada
}

Received: 7 May 2019 / Accepted: 1 June 2019 / Published online: 13 June 2019

(C) The Author(s) 2019

\begin{abstract}
We show how to calculate the effective potential of SU(3) QCD which tells that the true minimum is given by the monopole condensation. To do this we make the gauge independent Weyl symmetric Abelian decomposition of the SU(3) QCD which decomposes the gluons to the color neutral neurons and the colored chromons. In the perturbative regime this decomposes the Feynman diagram in such a way that the conservation of color is explicit. Moreover, this shows the existence of two gluon jets, the neuron jet and chromon jet, which can be verified by experiment. In the non-perturbative regime, the decomposition puts QCD to the background field formalism and reduces the non-Abelian gauge symmetry to a discrete color reflection symmetry, and provides us an ideal platform to calculate the one-loop effective action of QCD. Integrating out the chromons from the Weyl symmetric Abelian decomposition of QCD gauge invariantly imposing the color reflection invariance, we obtain the SU(3) QCD effective potential which generates the stable monopole condensation and the mass gap. We discuss the physical implications of our result, in particular the possible existence of the vacuum fluctuation mode of the monopole condensation in QCD.
\end{abstract}

\section{Introduction}

The color confinement problem in quantum chromodynamics (QCD) is one of the most challenging problems in theoretical physics. Two leading conjectures of the confinement mechanism are the monopole condensation [1-7] and the Abelian dominance [8,9]. The Abelian dominance asserts that only the Abelian (diagonal) part of the gluons is responsible for the color confinement. Intuitively this must be true, because the non-Abelian (off-diagonal) part describes the colored glu-

a e-mail: ymcho0416@gmail.com ons which are destined to be confined. Since the confined prisoner can not be the confining agent (the jailer), only the Abelian part can play the role of the confiner.

In fact we can prove this Abelian dominance rigorously. Theoretically we can show that the contribution of the nonAbelian part in the area law of the Wilson loop integral is negligible [9]. Moreover, numerically we can confirm this in the lattice QCD [10-15].

The problem with this conjecture is that this does not tell what is exactly the Abelian part of QCD, and how different is this from the Abelian gauge theory. More importantly, this does not tell how is the color confined by the Abelian part.

The monopole condensation tries to explain how is the color confined. It has long been argued that the confinement in QCD can be triggered by the monopole condensation. Indeed, if one assumes the monopole condensation, one could argue that the ensuing dual Meissner effect generates the color confinement [1-7]. Proving the monopole condensation, however, has been extremely difficult.

A natural way to establish the monopole condensation in QCD is to show that the quantum fluctuation triggers a phase transition similar to the dimensional transmutation observed in massless scalar QED [16]. There have been many attempts to demonstrate this. Savvidy first calculated the effective action of SU(2) QCD integrating out the colored gluons in the presence of an ad hoc color magnetic background, and has almost "proved" the magnetic condensation known as the Savvidy vacuum [17].

Unfortunately, the subsequent calculation repeated by Nielsen and Olesen showed that the effective action has an extra imaginary part which destabilizes the Savvidy vacuum. This is known as the "Savvidy-Nielsen-Olesen (SNO) instability" [18-26]. The origin of this instability can be traced to the tachyonic modes in the functional determinant of the gluon loop integral. 
But in physics we encounter tachyons when we do something wrong. For example, in spontaneous symmetry breaking we have tachyon when we choose the false vacuum. Similarly, in Neveu-Schwarz-Ramond (NSR) string theory we have the tachyonic vacuum when we do not make the theory modular invariant and supersymmetric with the GliozziScherk-Olive (GSO) projection [27-29]. So, obviously there is something wrong in the instability of the SNO vacuum. The question is how to remove the tachyonic modes in the gluon functional determinant, and how to justify that.

We emphasize, however, that the most serious defect of the SNO vacuum is not that it is unstable but that it is not gauge invariant. So even if the Savvidy vacuum were made stable, it can not be the QCD vacuum. Because of this Nielsen and Olesen has proposed the so-called "Copenhagen vacuum", the randomly oriented piecewise Savvidy vacuum [18-20]. But one can not obtain a gauge invariant vacuum simply by randomly orienting something which is not gauge invariant.

The gauge independent Abelian decomposition plays the crucial role to cure this defect. It decomposes QCD gauge potential to the color neutral Abelian part and colored valence part gauge independently. As importantly, it tells that the Abelian potential is made of two parts, the non-topological (Maxwellian) Abelian part and the topological (Diracian) monopole par [4-7]. This means that there are actually two possible magnetic backgrounds, the non-topological magnetic background (the Savvidy background) and topological monopole background. Moreover, we can show that only the monopole background is gauge invariant [30-34]. So choosing the monopole background in the calculation of the QCD effective action, we can avoid this trouble.

The Abelian decomposition also plays the crucial role to cure the SNO instability. It shows that after the Abelian decomposition the non-Abelian gauge symmetry is reduced to a simple discrete symmetry called the color reflection symmetry [4-7]. So the color reflection invariance assures the gauge invariance after the decomposition. This means that, integrating the colored gluons imposing this color reflection invariance under the monopole background we can calculate the QCD effective action gauge invariantly [30-34]. This removes the tachyonic modes and allows us to obtain the stable monopole condensation.

The fact that the monopole plays the crucial role in the color confinement is well established by now. First, using the Abelian decomposition we can prove that the Abelian part of the potential is responsible for the confining force in Wilson loop [9]. This, of course, is the Abelian dominance. But we can go further, and establish the monopole dominance theoretically as well as numerically. For instance, implementing the Abelian decomposition on lattice we can calculate Wilson loop contribution of the full potential, the Abelian potential, and the monopole potential separately, and show that all three potentials give the same area law [10-15]. This means that only the monopole potential is enough to generate the confining force.

The lattice results, however, does not tell how the monopole confines the color. In the preceding papers we have shown how to calculate the effective action of SU(2) QCD, and demonstrated that the stable monopole condensation can take place which generates the mass gap and color confinement in SU(2) QCD [30-34]. The purpose of this paper is to generalize this result to the real SU(3) QCD.

The crucial step to generalize the $\mathrm{SU}(2)$ result to $\mathrm{SU}(3)$ is to express the Abelian decomposition in SU(3) in the Weyl symmetric form. One might wonder how do we have the Weyl symmetric Abelian decomposition of SU(3) QCD, when we have only two Abelian directions in SU(3). The trick is to express the two Abelian potentials to three Abelian potentials of the SU(2) subgroups in Weyl symmetric way. This greatly simplifies for us to calculate the SU(3) QCD effective action from the SU(2) QCD effective action.

The paper is organized as follows. In Sect. 2 we review the Abelian decomposition of SU(2) QCD for later purpose. In Sect. 3 we discuss the Weyl symmetric Abelian decomposition of SU(3) QCD which greatly simplifies the calculation of the effective action of SU(3) QCD. In Sect. 4 we discuss the color reflection symmetry which replaces the role of the non-Abelian gauge symmetry after the Abelian decomposition, which plays a crucial role for us to implement the gauge invariance in the calculation of the QCD effective action. In Sect. 5 we repeat the calculation of the one-loop effective action of SU(2) QCD which plays the fundamental role for the SU(3) QCD effective action. In Sect. 6 we calculate SU(3) QCD effective action and demonstrate that the monopole condensation becomes the Weyl symmetric vacuum in SU(3) QCD. In particular we show that the essential features of SU(2) QCD, the dimensional transmutation by the monopole condensation which generates the mass gap remains the same. Finally in Sect. 7 we discuss the physical implications of our result.

\section{Abelian decomposition of SU(2) QCD: a review}

Before we discuss the Abelian decomposition we have to know why we need it. Consider the proton. The quark model tells that it is made of three quarks, but obviously we need gluon to bind them. On the other hand the quark model asserts that there is no "valence" gluon inside the proton which can be viewed as a constituent of proton. If so, what is the "binding" gluon inside the proton, and how do we distinguish it from the valence gluon?

Another motivation is the Abelian dominance. It has been believed that the Abelian part of gluon is responsible for the color confinement in QCD. As we have pointed out, this must be true. But what is the Abelian part, and how do we 
separate it? To answer these questions we need the Abelian decomposition.

Consider the SU(2) QCD for simplicity, and let $\left(\hat{n}_{1}, \hat{n}_{2}, \hat{n}_{3}\right.$ $=\hat{n}$ ) be an arbitrary right-handed local orthonormal basis. To make the Abelian decomposition we choose any direction, for example $\hat{n}$, to be the Abelian direction and impose the isometry to project out the restricted potential $\hat{A}_{\mu}$ [4-7]

$$
\begin{aligned}
D_{\mu} \hat{n} & =\left(\partial_{\mu}+g \vec{A}_{\mu} \times\right) \hat{n}=0 \\
\vec{A}_{\mu} \rightarrow \hat{A}_{\mu} & =A_{\mu} \hat{n}-\frac{1}{g} \hat{n} \times \partial_{\mu} \hat{n}=\mathcal{A}_{\mu}+\mathcal{C}_{\mu} \\
\mathcal{A}_{\mu} & =A_{\mu} \hat{n}, \quad \mathcal{C}_{\mu}=-\frac{1}{g} \hat{n} \times \partial_{\mu} \hat{n}, \quad A_{\mu}=\hat{n} \cdot \vec{A}_{\mu} .
\end{aligned}
$$

This is the Abelian projection which projects out the color neutral (i.e., Abelian) restricted potential.

We emphasize the followings. First, $\hat{A}_{\mu}$ is precisely the connection which leaves the Abelian direction invariant under the parallel transport (which makes $\hat{n}$ covariantly constant). Second, it is made of two parts, the non-topological (Maxwellian) $\mathcal{A}_{\mu}$ which describes the clor neutral gluon (the neuron) and the topological (Diracian) $\mathcal{C}_{\mu}$ which describes the non-Abelian monopole $[35,36]$. Third, the decomposition is gauge independent, because $\hat{n}$ is arbitrary. We can rotate $\hat{n}$ to any direction and still get exactly the same decomposition.

With this we have

$$
\begin{aligned}
\hat{F}_{\mu \nu} & =\left(F_{\mu \nu}+H_{\mu \nu}\right) \hat{n} \\
F_{\mu \nu} & =\partial_{\mu} A_{\nu}-\partial_{\nu} A_{\mu}, \\
H_{\mu \nu} & =-\frac{1}{g} \hat{n} \cdot\left(\partial_{\mu} \hat{n} \times \partial_{\nu} \hat{n}\right)=\partial_{\mu} C_{\nu}-\partial_{\nu} C_{\mu}, \\
C_{\mu} & =-\frac{1}{g} \hat{n}_{1} \cdot \partial_{\mu} \hat{n}_{2} .
\end{aligned}
$$

This tells the followings. First, $\hat{F}_{\mu \nu}$ has only the Abelian component. Second, $\hat{F}_{\mu \nu}$ is made of two potentials, the nontopological $A_{\mu}$ and topological $C_{\mu}$. This dual structure of $\hat{F}_{\mu \nu}$ plays an important role in the calculation of the QCD effective action, because this tells that there are actually two candidates of classical magnetic background to choose, the Savvidy background coming from $F_{\mu \nu}$ and the monopole background coming from $H_{\mu \nu}$ [30-34].

With (1) we can express the full SU(2) potential adding the non-Abelian (colored) part $\vec{X}_{\mu}$ [4-7]

$\vec{A}_{\mu}=\hat{A}_{\mu}+\vec{X}_{\mu}$,

$\vec{X}_{\mu}=\frac{1}{g} \hat{n} \times D_{\mu} \hat{n}, \quad \hat{n} \cdot \vec{X}_{\mu}=0$.

Under the infinitesimal gauge transformation

$\delta \vec{A}_{\mu}=\frac{1}{g} D_{\mu} \vec{\alpha}$

we have $\delta \hat{A}_{\mu}=\frac{1}{g} \hat{D}_{\mu} \vec{\alpha}, \quad\left(\hat{D}_{\mu}=\partial_{\mu}+g \hat{A}_{\mu} \times\right)$,

$\delta \vec{X}_{\mu}=-\vec{\alpha} \times \vec{X}_{\mu}$

This tells that $\hat{A}_{\mu}$ has the full SU(2) gauge degrees of freedom, even though it is restricted. Moreover, $\vec{X}_{\mu}$ becomes gauge covariant. This is a direct consequence of the fact that the connection space (the space of all potentials) forms an affine space. With this $\vec{X}_{\mu}$ can be interpreted to describe the colored gluon (the chromon). This is the Abelian decomposition which decomposes the $\mathrm{SU}(2)$ gluons to one Abelian neuron and two colored chromons gauge independently. Notice that we can express the chromon by $\vec{R}_{\mu}$ indicating the color, or in the complex notation by $R_{\mu}$ with $R_{\mu}=$ $\left(X_{\mu}+i X_{\mu}^{2}\right) / \sqrt{2}$.

This should be compared with the popular Abelian decomposition based on Maximal Abelian Gauge (MAG) condition [8]. Here the decomposition is given by $\vec{A}_{\mu}=A_{\mu} \hat{n}+\check{X}_{\mu}$ with $\check{X}_{\mu}=A_{\mu}^{1} \hat{n}_{1}+A_{\mu}^{2} \hat{n}_{2}$, with the MAG condition $\check{D}_{\mu} \check{X}_{\mu}=0$. So the neuron and the chromon are described by the Abelian (diagonal) component and the non-Abelian (off-diagonal) component of $\vec{A}_{\mu}$.

But obviously this decomposition is not gauge independent, and the chromon $\check{X}_{\mu}$ does not transform covariantly. Worse, the topology of the non-Abelian gauge symmetry which plays the crucial role in QCD is completely neglected in this decomposition. In contrast the topological part plays an essential role in our Abelian decomposition. In fact (3) tells that the Abelian decomposition is made of three parts, the neuron, chromon, and the topological parts. Without the topological part we can not decompose the gluon to neuron and chromon gauge independently.

With the restricted potential we can construct the restricted QCD (RCD) which has the full non-Abelian gauge symmetry but is simpler than the QCD

$$
\begin{aligned}
\mathcal{L}_{R C D}= & -\frac{1}{4} \hat{F}_{\mu \nu}^{2}=-\frac{1}{4} F_{\mu \nu}^{2} \\
& +\frac{1}{2 g} F_{\mu \nu} \hat{n} \cdot\left(\partial_{\mu} \hat{n} \times \partial_{\nu} \hat{n}\right)-\frac{1}{4 g^{2}}\left(\partial_{\mu} \hat{n} \times \partial_{\nu} \hat{n}\right)^{2},
\end{aligned}
$$

which describes the Abelian sub-dynamics of QCD. Since RCD contains the non-Abelian monopole degrees explicitly, it provides an ideal platform for us to study the monopole physics gauge independently.

From (3) we have

$\vec{F}_{\mu \nu}=\hat{F}_{\mu \nu}+\hat{D}_{\mu} \vec{X}_{v}-\hat{D}_{\nu} \vec{X}_{\mu}+g \vec{X}_{\mu} \times \vec{X}_{\nu}$

With this we can express QCD by

$$
\begin{aligned}
\mathcal{L}_{E C D}= & -\frac{1}{4} \vec{F}_{\mu \nu}^{2}=-\frac{1}{4} \hat{F}_{\mu \nu}^{2}-\frac{1}{4}\left(\hat{D}_{\mu} \vec{X}_{\nu}-\hat{D}_{\nu} \vec{X}_{\mu}\right)^{2} \\
& -\frac{g}{2} \hat{F}_{\mu \nu} \cdot\left(\vec{X}_{\mu} \times \vec{X}_{\nu}\right)-\frac{g^{2}}{4}\left(\vec{X}_{\mu} \times \vec{X}_{\nu}\right)^{2}
\end{aligned}
$$




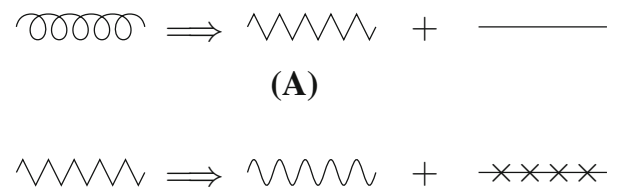

(B)

Fig. 1 The Abelian decomposition of the gauge potential. In a it is decomposed to the restricted potential (kinked line) and the valence potential (straight line) which describes the chromon. In $\mathbf{b}$ the restricted potential is further decomposed to the Maxwell part (wiggly line) which describes the neuron and the Dirac part (spiked line) which describes the monopole

This is the extended QCD (ECD) which shows that QCD can be viewed as RCD which has the chromon as the colored source [4-7].

We can easily add the quark in the Abelian decomposition,

$$
\begin{aligned}
\mathcal{L}_{q} & =\sum_{k} \bar{\Psi}_{k}\left(i \gamma^{\mu} D_{\mu}-m\right) \Psi_{k} \\
& =\sum_{k}\left[\bar{\Psi}_{k}\left(i \gamma^{\mu} \hat{D}_{\mu}-m\right) \Psi_{k}+\frac{g}{2} \vec{X}_{\mu} \cdot \bar{\Psi}_{k}\left(\gamma^{\mu} \vec{\tau}\right) \Psi_{k}\right], \\
\hat{D}_{\mu} & =\partial_{\mu}+\frac{g}{2 i} \vec{\tau} \cdot \hat{A}_{\mu},
\end{aligned}
$$

where $\Psi$ is the quark doublet, $m$ is the mass, and $k$ is the flavour index.

Mathematically ECD is identical to QCD, but physically it provides a totally new meaning to QCD. It tells that QCD has two types of gluons, neuron and chromon, which plays different roles. The neuron, together with the topological monopole potential, provides the binding. On the other hand the chromon, just like the quarks, becomes the colored source which is destined to be confined [4-7,34].

We can express the Abelian decomposition graphically. This is shown in Fig. 1, where the gauge potential is decomposed to the restricted potential which has the full gauge degrees of freedom and the gauge covariant valence potential which describes the chromon in (a), and the restricted potential is decomposed further to the non-topological Maxwell part $\mathcal{A}_{\mu}$ which describes the neuron and the topological Dirac part $\mathcal{C}_{\mu}$ which describes the monopole in (b).

A direct consequence of the Abelian decomposition is the decomposition of the Feynman diagram. Clearly (7) and (8) tells that the SU(2) QCD vertices can be decomposed to the neuron and chromon interaction. This is shown in Fig. 2. Notice that the conservation of color forbids the three-point gluon vertex made of three neurons or three chromons. Similarly, the four-point gluon vertex made of four neurons or one neuron and three chromons is forbidden. This is because the SU(2) QCD has only one color. Without the Abelian decomposition this would have been impossible.

At this point one might wonder why the monopole part does not appear in the Feynman diagram. There has been an

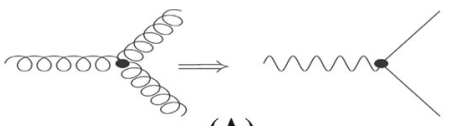

(A)
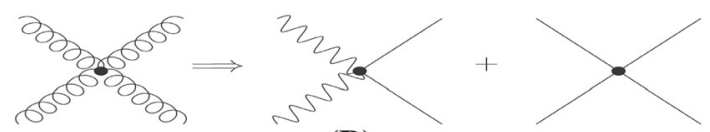

(B)

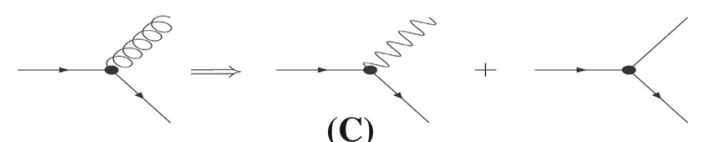

(C)

Fig. 2 The decomposition of the Feynman diagrams in SU(2) QCD. The three-point and four-point gluon vertices are decomposed in a and $\mathbf{b}$, and the quark-gluon vertices are decomposed in c. Notice that since the monopole is not a propagating degree it does not appear in the Feynman diagram

assertion in the literature that the introduction of the Abelian direction $\hat{n}$ adds a new dynamical degree, and thus alters QCD $[37,38]$. This is a gross misunderstanding of the Abelian decomposition. As we have emphasized, we can change $\hat{n}$ to any direction we like by a gauge transformation, for example to a trivial configuration $(0,0,1)$. This tells that $\hat{n}$ does not represent a dynamical degree, but a gauge degree. In fact, we can show explicitly that $\hat{n}$ has no equation of motion to satisfy, so that it can not describe a propagating degree [39]. This is why the monopole part does not appear in the Feynman diagram.

This does not mean that $\hat{n}$ is not important. In fact it plays a very important role, since the non-Abelian gauge degrees of QCD has the non-trivial topological structure which could change the physics drastically. Indeed $\hat{n}$ here represents not only the monopole topology $\pi_{2}\left(S^{2}\right)$ but also the vacuum topology $\pi_{3}\left(S^{3}\right) \simeq \pi_{3}\left(S^{2}\right)$ of the $\mathrm{SU}(2)$ gauge theory, both of which are the essential characteristics of QCD $[35,40]$.

Moreover, it allows us to make the Abelian decomposition and prove that there are two types of gluons which play totally different roles gauge independently. Most importantly it provides an ideal platform for us to calculate the QCD effective action and prove the monopole condensation. This would have been impossible without it. In the literature the Abelian decomposition has been referred to the Cho decomposition, Cho-Duan-Ge (CDG) decomposition, or Cho-Faddeev-Niemi (CFN) decomposition [10$13,37,38,41-45]$.

\section{Weyl symmetric abelian decomposition of SU(3) QCD}

The Abelian decomposition of SU(3) QCD is technically a bit more complicated but straightforward [46-48]. Since SU(3) has rank two, we have two Abelian subgroups of SU(3). So 
we need two Abelian directions to make the Abelian decomposition.

Let $\hat{n}_{i}(i=1,2, \ldots, 8)$ be the local orthonormal SU(3) basis. Clearly we can choose the Abelian directions to be $\hat{n}_{3}=\hat{n}$ and $\hat{n}_{8}=\hat{n}^{\prime}$. Now make the Abelian projection by

$D_{\mu} \hat{n}=0$.

This automatically guarantees [35]

$D_{\mu} \hat{n}^{\prime}=0, \quad \hat{n}^{\prime}=\frac{1}{\sqrt{3}} \hat{n} * \hat{n}$.

where $*$ denotes the $d$-product. This is because $\mathrm{SU}(3)$ has two vector products, the anti-symmetric $f$-product and the symmetric $d$-product. This tells that we actually need only one $\lambda_{3}$-like Abelian direction to make the Abelian decomposition, although $\mathrm{SU}(3)$ has two Abelian directions.

Solving (9), we have the following Abelian projection which projects out the Abelian restricted potential,

$$
\begin{aligned}
\vec{A}_{\mu} \rightarrow \hat{A}_{\mu} & =A_{\mu} \hat{n}+A_{\mu}^{\prime} \hat{n}^{\prime}-\frac{1}{g} \hat{n} \times \partial_{\mu} \hat{n}-\frac{1}{g} \hat{n}^{\prime} \times \partial_{\mu} \hat{n}^{\prime} \\
& =\sum_{p} \frac{2}{3} \hat{A}_{\mu}^{p}, \quad(p=1,2,3), \\
\hat{A}_{\mu}^{p} & =A_{\mu}^{p} \hat{n}^{p}-\frac{1}{g} \hat{n}^{p} \times \partial_{\mu} \hat{n}^{p}=\mathcal{A}_{\mu}^{p}+\mathcal{C}_{\mu}^{p}, \\
A_{\mu}^{1} & =A_{\mu}, \quad A_{\mu}^{2}=-\frac{1}{2} A_{\mu}+\frac{\sqrt{3}}{2} A_{\mu}^{\prime}, \\
A_{\mu}^{3} & =-\frac{1}{2} A_{\mu}-\frac{\sqrt{3}}{2} A_{\mu}^{\prime}, \quad \hat{n}^{1}=\hat{n}, \\
\hat{n}^{2} & =-\frac{1}{2} \hat{n}+\frac{\sqrt{3}}{2} \hat{n}^{\prime}, \quad \hat{n}^{3}=-\frac{1}{2} \hat{n}-\frac{\sqrt{3}}{2} \hat{n}^{\prime},
\end{aligned}
$$

where the sum is the sum of the three Abelian directions $\left(\hat{n}^{1}, \hat{n}^{2}, \hat{n}^{3}\right)$ of three $\mathrm{SU}(2)$ subgroups made of $\left(\hat{n}_{1}, \hat{n}_{2}, \hat{n}^{1}\right)$, $\left(\hat{n}_{6}, \hat{n}_{7}, \hat{n}^{2}\right),\left(\hat{n}_{4}, \hat{n}_{5}, \hat{n}^{3}\right) .{ }^{1}$ Notice the factor $2 / 3$ in front of $\hat{A}_{\mu}^{p}$ in the $p$-summation. This is because the three SU(2) restricted potentials are not independent.

What is remarkable about the above expression is that, although SU(3) has two Abelian directions, the restricted potential is expressed by the restricted potentials of three SU(2) subgroups in a Weyl symmetric way. The Weyl symmetry of SU(3) is given by the six element permutation group of three SU(2) subgroups, or equivalently three colors of $\mathrm{SU}(3)$.

In general the Weyl group of SU(N) is the $N$ ! elements permutation group of $N$ colors of $\mathrm{SU}(\mathrm{N})$, which is mathematically identical to the symmetric group $S_{N}$ of order $N$. And we can show that the restricted potential of the SU(N) QCD can be expressed by the sum of the restricted potentials

\footnotetext{
1 Our notation of $\lambda$-matrices is the same as the GellMann's notation, except that our $\lambda_{5}$ has the opposite signature. Our notation is more convenient for the Weyl symmetry.
}

of $N$ SU(2) subgroups in a Weyl symmetric way. This is very important because, as we will see, this allows us to calculate the SU(N) QCD effective action in terms of the SU(2) QCD effective action.

Just as in the SU(2) QCD we can easily show that the $\mathrm{SU}(3)$ restricted potential has the full gauge degrees of freedom, and construct the SU(3) RCD made of the restricted field strength,

$\mathcal{L}_{R C D}=-\sum_{p} \frac{1}{6}\left(\hat{F}_{\mu \nu}^{p}\right)^{2}$,

which has the full SU(3) gauge symmetry. Here again the factor $1 / 6$ comes from the fact that the three $\mathrm{SU}(2)$ restricted field strengths are not independent.

With (11) we have the Abelian decomposition of the SU(3) gauge potential,

$$
\begin{aligned}
\vec{A}_{\mu} & =\hat{A}_{\mu}+\vec{X}_{\mu}=\sum_{p}\left(\frac{2}{3} \hat{A}_{\mu}^{p}+\vec{W}_{\mu}^{p}\right), \\
\vec{X}_{\mu} & =\sum_{p} \vec{W}_{\mu}^{p}, \\
\vec{W}_{\mu}^{1} & =X_{\mu}^{1} \hat{n}_{1}+X_{\mu}^{2} \hat{n}_{2}, \quad \vec{W}_{\mu}^{2}=X_{\mu}^{6} \hat{n}_{6}+X_{\mu}^{7} \hat{n}_{7}, \\
\vec{W}_{\mu}^{3} & =X_{\mu}^{4} \hat{n}_{4}+X_{\mu}^{5} \hat{n}_{5} .
\end{aligned}
$$

Here again $\vec{X}_{\mu}$ transforms covariantly. Moreover, it can be decomposed to the three chromons $\vec{W}_{\mu}^{p}$ of the $\mathrm{SU}(2)$ subgroups. So we have two neurons and six (or three complex) chromons in SU(3) QCD. And we can identify $\left(\vec{W}_{\mu}^{1}, \vec{W}_{\mu}^{2}, \vec{W}_{\mu}^{3}\right)$ as the red, blue, and green chromons $\left(\vec{R}_{\mu}, \vec{B}_{\mu}, \vec{G}_{\mu}\right)$, or equivalently by $\left(R_{\mu}, B_{\mu}, G_{\mu}\right)$ with

$$
\begin{aligned}
R_{\mu} & =\frac{X_{\mu}^{1}+i X_{\mu}^{2}}{\sqrt{2}}, \quad B_{\mu}=\frac{X_{\mu}^{6}+i X_{\mu}^{7}}{\sqrt{2}} \\
G_{\mu} & =\frac{X_{\mu}^{4}+i X_{\mu}^{5}}{\sqrt{2}}
\end{aligned}
$$

in the complex notation.

From (13) we have

$$
\begin{aligned}
\hat{D}_{\mu} \vec{X}_{v} & =\sum_{p} \hat{D}_{\mu}^{p} \vec{W}_{\nu}^{p}, \quad \hat{D}_{\mu}^{p}=\partial_{\mu}+g \hat{A}_{\mu}^{p} \times, \\
\vec{X}_{\mu} \times \vec{X}_{v} & =\sum_{p, q} \vec{W}_{\mu}^{p} \times \vec{W}_{\nu}^{q},
\end{aligned}
$$

so that

$$
\begin{aligned}
\vec{F}_{\mu \nu}= & \hat{F}_{\mu \nu}+\hat{D}_{\mu} \vec{X}_{\nu}-\hat{D}_{\nu} \vec{X}_{\mu}+g \vec{X}_{\mu} \times \vec{X}_{\nu} \\
= & \sum_{p}\left[\frac{2}{3} \hat{F}_{\mu \nu}^{p}+\left(\hat{D}_{\mu}^{p} \vec{W}_{\nu}^{p}-\hat{D}_{\mu}^{p} \vec{W}_{\nu}^{p}\right)\right] \\
& +\sum_{p, q} \vec{W}_{\mu}^{p} \times \vec{W}_{\nu}^{q} .
\end{aligned}
$$




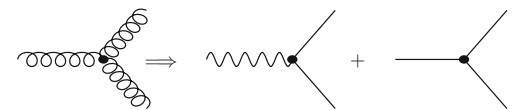

(A)

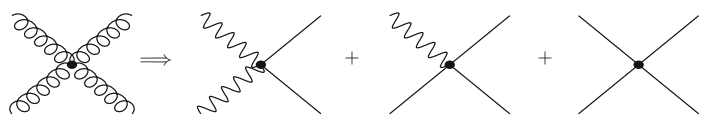

(B)

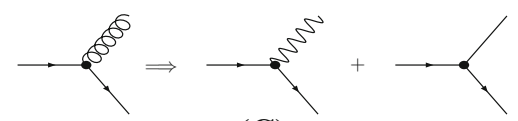

(C)

Fig. 3 The decomposition of the Feynman diagrams in SU(3) QCD. The three-point and four-point gluon vertices are decomposed in $\mathbf{a}$ and $\mathbf{b}$, and the quark-gluon vertices are decomposed in c. Notice the difference between SU(2) QCD and SU(3) QCD

With this we have the following form of SU(3) ECD [46]

$$
\begin{aligned}
\mathcal{L}_{E C D}= & -\frac{1}{4} \vec{F}_{\mu \nu}^{2}=\sum_{p}\left\{-\frac{1}{6}\left(\hat{F}_{\mu \nu}^{p}\right)^{2}\right. \\
& \left.-\frac{1}{4}\left(\hat{D}_{\mu}^{p} \vec{W}_{\nu}^{p}-\hat{D}_{\nu}^{p} \vec{W}_{\mu}^{p}\right)^{2}-\frac{g}{2} \hat{F}_{\mu \nu}^{p} \cdot\left(\vec{W}_{\mu}^{p} \times \vec{W}_{\nu}^{p}\right)\right\} \\
& -\sum_{p, q} \frac{g^{2}}{4}\left(\vec{W}_{\mu}^{p} \times \vec{W}_{\mu}^{q}\right)^{2} \\
& -\sum_{p, q, r} \frac{g}{2}\left(\hat{D}_{\mu}^{p} \vec{W}_{\nu}^{p}-\hat{D}_{\nu}^{p} \vec{W}_{\mu}^{p}\right) \cdot\left(\vec{W}_{\mu}^{q} \times \vec{W}_{\mu}^{r}\right) \\
& -\sum_{p \neq q} \frac{g^{2}}{4}\left[\left(\vec{W}_{\mu}^{p} \times \vec{W}_{\nu}^{q}\right) \cdot\left(\vec{W}_{\mu}^{q} \times \vec{W}_{\nu}^{p}\right)\right. \\
& \left.+\left(\vec{W}_{\mu}^{p} \times \vec{W}_{\nu}^{p}\right) \cdot\left(\vec{W}_{\mu}^{q} \times \vec{W}_{\nu}^{q}\right)\right],
\end{aligned}
$$

which puts QCD to a totally different expression.

We can add quarks in the Abelian decomposition,

$$
\begin{aligned}
\mathcal{L}_{q} & =\bar{\Psi}\left(i \gamma^{\mu} D_{\mu}-m\right) \Psi \\
& =\bar{\Psi}\left(i \gamma^{\mu} \hat{D}_{\mu}-m\right) \Psi+\frac{g}{2} \vec{X}_{\mu} \cdot \bar{\Psi}\left(\gamma^{\mu} \vec{t}\right) \Psi \\
& =\sum_{p}\left[\bar{\Psi}^{p}\left(i \gamma^{\mu} \hat{D}_{\mu}^{p}-m\right) \Psi^{p}+\frac{g}{2} \vec{W}_{\mu}^{p} \cdot \bar{\Psi}^{p}\left(\gamma^{\mu} \vec{\tau}^{p}\right) \Psi^{p}\right], \\
\hat{D}_{\mu} & =\partial_{\mu}+\frac{g}{2 i} \vec{t} \cdot \hat{A}_{\mu}, \hat{D}_{\mu}^{p}=\partial_{\mu}+\frac{g}{2 i} \vec{\tau}^{p} \cdot \hat{A}_{\mu}^{p},
\end{aligned}
$$

where $m$ is the mass, $p$ denotes the color of the quarks, and $\Psi^{p}$ represents the three $\mathrm{SU}(2)$ quark doublets (i.e., $(r, b)$, $(b, g)$, and $(g, r)$ doublets) of the $(r, b, g)$ quark triplet. Notice that here we have suppressed the flavour degrees.

As we have emphasized, ECD does not change QCD. However, it reveals the important hidden characteristics of QCD and make them clearly visible. In the perturbative regime it refines the Feynman diagrams drastically. The decomposition of the Feynman diagram of the SU(3) QCD is shown graphically in Fig. 3. In (a) the three-point QCD gluon vertex is decomposed to two vertices, the one made of

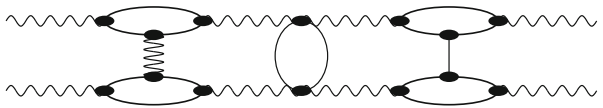

(A)

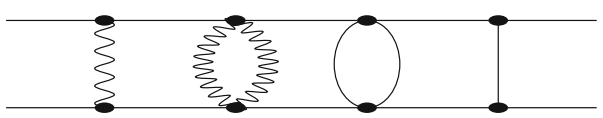

(B)

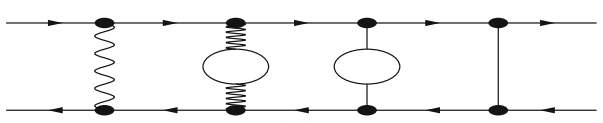

(C)

Fig. 4 The possible Feynman diagrams of the neuron and chromon interactions. Two neuron interaction is shown in a, two chromon interaction is shown in $\mathbf{b}$, and quark-antiquark interaction is shown in $\mathbf{c}$

one neuron and two chromons and the other made of three chromons. In (b) the four-point gluon vertex is decomposed to three vertices made of one neuron and three chromons, two neurons and two chromons, and four chromons. In (c) the quark-gluon vertex is decomposed to the quark-neuron vertex and quark-chromon vertex. Obviously this decomposition of the Feynman diagrams is not possible in the conventional QCD where all gluons are treated equally.

There are two points to be emphasized here. First, the diagrams do not contain the monopole. As we have already pointed out, this is because $\hat{n}$ represents the topological degree. Another reason why the monopole does not appear in the Feynman diagram is that the monopole makes the condensation, so that it disappears after the confinement sets in. So in the perturbative regime (inside the hadrons) only the neurons and chromons contribute to the Feynman diagrams.

Second, the conservation of the color is explicit in the diagrams. For example, the three-point vertex made of three neurons or two neurons and one chromon, and the four-point vertex made of three or four neurons are forbidden by the conservation of color. Moreover, the quark-neuron interaction does not change the quark color, but the quark-chromon interaction changes the quark color.

This decomposition of the Feynman diagram should play important roles in the perturbative QCD. With this we can pinpoint what diagrams are actually responsible for the coupling constant renormalization and asymptotic freedom. Moreover, it allows us to demonstrate graphically that the neurons and chromons play totally different roles. To see this consider the three Feynman diagrams which describe the interaction of two neurons, two chromons, and quarkantiquark, shown in Fig. 4.

Clearly the neuron interaction in (a) looks exactly like the two photon interaction in QED. This is because the neurons are color neutral, so that they behave like the photons in QED. On the other hand, the chromon interaction in (b) looks 
exactly like the quark interaction shown in (c). Again this is because the chromons behave as colored source. The contrast between the neuron and chromon interactions is unmistakable.

This has a deep implication in hadron spectroscopy. This means that the chromons can become the constituent of hadrons, so that they, just like the quarks, can form hadronic bound states which can be identified as the glueballs. Moreover, together with quarks they could form new hybrid baryons. But the neuron binding shown in (a) strongly implies that they can hardly make a bound state, which suggests that the neurons may not become the constituent of hadrons. This leads us to generalize the quark model to the quark and chromon model, which could provide a new picture of hadrons $[47,48]$.

But the Abelian decomposition plays the decisive role in the non-perturbative regime. First of all, it allows us to prove not only the Abelian dominance but also the monopole dominance in QCD rigorously. Obviously the chromons can not play any role in the confinement, because they themselves have to be confined. This can be provided theoretically. First, we can show that only the restricted potential contributes to the Wilson loop integral which generates the linear confining potential [30,31]. This is the Abelian dominance.

Moreover, we can argue that actually the monopole part of the restricted potential is responsible for the confining potential. This is because the Maxwell part plays the role of the electromagnetic potential in QED, which is known to have no confinement. This demonstrates the monopole dominance, that the monopole is responsible for the confinement.

This is backed up numerically in the lattice QCD. Implementing the Abelian decomposition on the lattice, we can show that the confining force comes from the monopole part of the restricted potential [10-15]. The recent result of SU(3) lattice calculation is shown in Fig. 5, which clearly tells that all three potentials, the full potential, the Abelian potential, and the monopole potential generate the same confining force. This establishes the monopole dominance.

The monopole dominance, however, does not tell us how the monopole confines the color. Fortunately, the Abelian decomposition allows us to show how. To see this it is important to understand that field theoretically ECD puts QCD in the background field formalism [39,49-51]. This is because in ECD the restricted potential and the valence potential can be treated as the slow varying classical field and the fluctuating quantum field.

This enlarges the gauge symmetry of QCD and makes ECD to have two independent color gauge symmetries, the classical and quantum gauge symmetry. This has a deep consequence. For example, the quantum gauge symmetry keeps the chromons massless, even though they transform gauge covariantly [39].

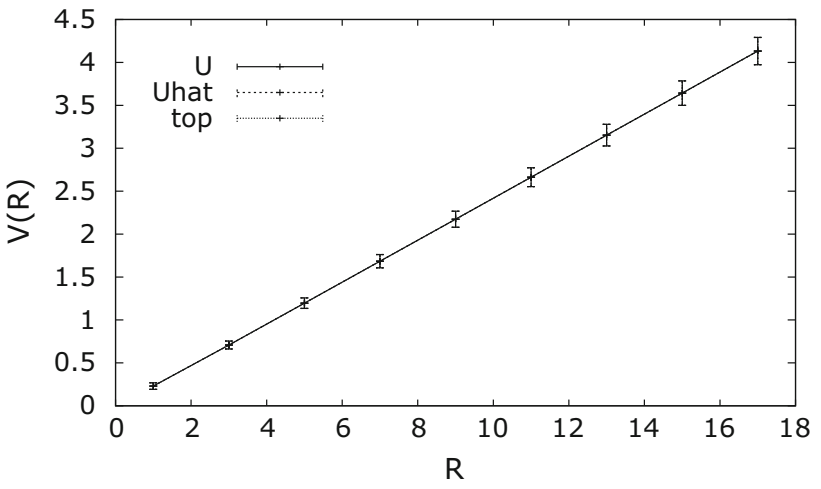

Fig. 5 The SU(3) lattice QCD calculation which establishes the monopole dominance in the confining force in Wilson loop. Here the confining forces shown in full, dashed, and dotted lines are obtained with the full potential, the Abelian potential, and the monopole potential, respectively

More importantly, the background field formalism provides us an ideal platform to calculate the QCD effective action gauge independently. This is because we can treat the slow varying classical part as the background, and integrate the quantum part to obtain the one-loop effective action.

Furthermore, ECD simplifies the complicated non-Abelian gauge symmetry to a simple discrete symmetry called the color reflection symmetry which is much easier to handle [4-7]. This plays a crucial role for us to implement the gauge invariance when we calculate the QCD effective action to demonstrate the monopole condensation, and allows us to clarify the confusions of the old calculations of the effective action $[34,46]$.

But most importantly the Abelian decomposition allows us to calculate the SU(3) QCD effective action directly from the SU(2) QCD effective action [46]. This is because the Abelian decomposition of SU(3) QCD transforms it to a Weyl symmetric form of three SU(2) QCD. This is evident in (16) and (17), which tell that SU(3) QCD is Weyl symmetric, symmetric under the permutation of three SU(2) subgroups. As we will see, this Weyl symmetry greatly simplifies the calculation of the SU(3) QCD effective action. In general we can show that the Weyl symmetric Abelian decomposition of SU(N) QCD allows us to obtain the SU(N) QCD effective action directly from the SU(2) QCD effective action.

\section{Color reflection invariance}

As we have emphasized, the Abelian decomposition is gauge independent. On the other hand, the selection of the Abelian direction amounts to the gauge fixing. So, once we fix the Abelian direction the gauge symmetry is broken. On the other hand, this does not break the gauge symmetry completely, so that we have a residual discrete symmetry called the color 
reflection symmetry even after the Abelian decomposition [4-7,34].

The importance of this residual symmetry comes from the following observation. First, this plays the role of the gauge symmetry after the Abelian decomposition. Second, this symmetry is much simpler than the color gauge symmetry. This tells that the Abelian decomposition reduces the complicated non-Abelian gauge symmetry to a simple discrete symmetry which is much easier to handle. This greatly helps us to implement the gauge invariance in the calculation of the QCD effective action. So we discuss the color reflection symmetry first.

Consider the SU(2) QCD first and make the color reflection, the $\pi$-rotation of the $\mathrm{SU}(2)$ basis along the $\hat{n}_{2}$-direction which inverts the color direction $\hat{n}$,

$\left(\hat{n}_{1}, \hat{n}_{2}, \hat{n}\right) \rightarrow\left(-\hat{n}_{1}, \hat{n}_{2},-\hat{n}\right)$.

Obviously this is a gauge transformation which should not change the physics. On the other hand, under the color reflection (18) we have [34]

$\hat{A}_{\mu} \rightarrow \hat{A}_{\mu}^{(c)}=-A_{\mu} \hat{n}-\frac{1}{g} \hat{n} \times \partial_{\mu} \hat{n}$,

$A_{\mu} \rightarrow A_{\mu}^{(c)}=-\hat{n} \cdot \vec{A}_{\mu}=-A_{\mu}$.

Moreover,

$\vec{X}_{\mu} \rightarrow \vec{X}_{\mu}^{(c)}=-\left(X_{\mu}^{1} \hat{n}_{1}-X_{\mu}^{2} \hat{n}_{2}\right)$,

or, in the complex notation by

$R_{\mu} \rightarrow R_{\mu}^{(c)}=-\bar{R}_{\mu}$,

where $\bar{R}_{\mu}=\left(X_{\mu}-i X_{\mu}^{2}\right) / \sqrt{2}$.

But since the isometry condition (1) is insensitive to (18), we have two different Abelian decompositions imposing the same isometry,

$\vec{A}_{\mu}=\hat{A}_{\mu}+\vec{X}_{\mu}, \quad \vec{A}_{\mu}=\hat{A}_{\mu}^{(c)}+\vec{X}_{\mu}^{(c)}$,

without changing the physics. This is why the color reflection (18) becomes a discrete symmetry of QCD after the Abelian decomposition [4-7].

To understand the meaning of this, notice that the neuron potential $A_{\mu}$ change the signature, while the topological part remains invariant. Moreover the chromon changes to the complex conjugate partner (together with the change of the signature), which changes the chromon to anti-chromon and flips the sign of the chromon charge.

This is not surprising. In the absence of the topological part (7) describes QED which is coupled to the massless charged vector field where the neuron plays the role of the photon. And in QED it is well known that the photon has negative charge conjugation quantum number. So it is natural that $A_{\mu}$ in SU(2) QCD changes the signature under the color reflec- tion. Similarly we can argue that $A_{\mu}$ changes the signature under the parity [34].

On the other hand the monopole potential remains unchanged under the color reflection. This means that the monopole and anti-monopole are physically undistinguishable in QCD $[4,5,36]$. This should be contrasted with the monopole in spontaneously broken gauge theories, where the monopole and ant-monopole are physically different.

This confirms that, although there are two possible magnetic backgrounds, only the monopole background coming from $\mathcal{C}_{\mu}$ is qualified to be the legitimate background we can choose in the calculation of the QCD effective action. This is because $A_{\mu}$ changes the signature under the color reflection and thus fails to be gauge invariant. Indeed this is the reason why the Savvidy vacuum is not gauge invariant.

As importantly, (20) tells that the physics should not change when we change the chromon to anti-chromon. In fact (20) tells that the chromon and anti-chromon are the color reflection partner. This means that they can not be separately discussed in QCD and should always play exactly the same amount of role. This is the reason why the color should become unphysical and confined, which makes QCD totally different from QCD. This point plays a crucial role when we implement the gauge invariance in the calculation of the effective action [4-7,34]

In the fundamental representation the color reflection (18) is given by the 4 element subgroup of SU(2) made of [4-7]

$$
\begin{aligned}
& C_{1}=\left(\begin{array}{ll}
1 & 0 \\
0 & 1
\end{array}\right), \quad C_{2}=\left(\begin{array}{cc}
-1 & 0 \\
0 & -1
\end{array}\right), \\
& C_{3}=\left(\begin{array}{cc}
0 & 1 \\
-1 & 0
\end{array}\right), \quad C_{4}=\left(\begin{array}{cc}
0 & -1 \\
1 & 0
\end{array}\right) .
\end{aligned}
$$

This can be expressed by

$C_{k}=D_{a} R_{b}, \quad(a=1,2 ; b=1,2 ; k=1,2, \ldots, 4)$,

$D_{1}=\left(\begin{array}{ll}1 & 0 \\ 0 & 1\end{array}\right), \quad D_{2}=\left(\begin{array}{cc}-1 & 0 \\ 0 & -1\end{array}\right)$

$R_{1}=\left(\begin{array}{ll}1 & 0 \\ 0 & 1\end{array}\right), \quad R_{2}=\left(\begin{array}{cc}0 & 1 \\ -1 & 0\end{array}\right)$,

which contains the diagonal subgroup made of $D_{1}$ and $D_{2}$. This becomes the residual symmetry of the SU(2) quark doublet $(r, b)$ after the Abelian decomposition. Notice that $R_{2}$ plays the role of the generator of the color reflection group.

As for the gluons which form the adjoint representation the color reflection can be simplified further for the following reasons. First, the diagonal subgroup has no effect on the adjoint representation. Second, the color reflection changes $\hat{n}$ to $-\hat{n}$ and $\left(\hat{n}_{1}, \hat{n}_{2}\right)$ to $\left(-\hat{n}_{1}, \hat{n}_{2}\right)$. So, the gluon triplet is decomposed to two independent representations.

Indeed, for the neuron we have

$R_{2}: A_{\mu} \rightarrow-A_{\mu}$. 
But for the chromon we have

$R_{2}:\left(\vec{X}_{\mu}, \vec{X}_{\mu}^{(c)}\right) \rightarrow-\left(\vec{X}_{\mu}^{(c)}, \vec{X}_{\mu}\right)$,

or equivalently

$R_{2}:\left(R_{\mu}, \bar{R}_{\mu}\right) \rightarrow-\left(\bar{R}_{\mu}, R_{\mu}\right)$.

This confirms that the neuron and chromon transform independently, forming one-dimensional and two-dimensional representations under the color reflection. This drastically simplifies the non-Abelian gauge symmetry.

For SU(3) the fundamental representation the color reflection group is made of 24 elements subgroup of SU(3) given by $[4-7,45]$

$$
\begin{aligned}
& C_{k}=D_{a} R_{b} \text {, } \\
& (a=1,2,3,4 ; b=1,2, \ldots, 6 ; k=1,2, \ldots, 24), \\
& D_{1}=\left(\begin{array}{lll}
1 & 0 & 0 \\
0 & 1 & 0 \\
0 & 0 & 1
\end{array}\right), \quad D_{2}=\left(\begin{array}{ccc}
-1 & 0 & 0 \\
0 & -1 & 0 \\
0 & 0 & 1
\end{array}\right) \text {, } \\
& D_{3}=\left(\begin{array}{ccc}
1 & 0 & 0 \\
0 & -1 & 0 \\
0 & 0 & -1
\end{array}\right), D_{4}=\left(\begin{array}{ccc}
-1 & 0 & 0 \\
0 & 1 & 0 \\
0 & 0 & -1
\end{array}\right) \text {, } \\
& R_{1}=\left(\begin{array}{lll}
1 & 0 & 0 \\
0 & 1 & 0 \\
0 & 0 & 1
\end{array}\right), \quad R_{2}=\left(\begin{array}{ccc}
0 & 1 & 0 \\
-1 & 0 & 0 \\
0 & 0 & 1
\end{array}\right) \text {, } \\
& R_{3}=\left(\begin{array}{ccc}
1 & 0 & 0 \\
0 & 0 & 1 \\
0 & -1 & 0
\end{array}\right), \quad R_{4}=\left(\begin{array}{ccc}
0 & 0 & 1 \\
0 & -1 & 0 \\
1 & 0 & 0
\end{array}\right) \text {, } \\
& R_{5}=\left(\begin{array}{lll}
0 & 1 & 0 \\
0 & 0 & 1 \\
1 & 0 & 0
\end{array}\right), R_{6}=\left(\begin{array}{ccc}
0 & 0 & 1 \\
-1 & 0 & 0 \\
0 & -1 & 0
\end{array}\right) \text {, }
\end{aligned}
$$

where the four $D$-matrices form the diagonal subgroup. This describes the residual symmetry of the quark triplet $(r, b, g)$ after the Abelian decomposition. Notice that here $R_{2}$ and $R_{3}$ play the role of the generator. For example, we have $R_{5}=$ $R_{3} \cdot R_{2}, R_{6}=R_{2} \cdot R_{3}$, and $R_{4}=R_{2} \cdot R_{3} \cdot R_{2}$.

For the gluon octet which form the adjoint representation of SU(3) the color reflection can be simplified further. Just as in SU(2) QCD, the neurons and chromons transform separately, among themselves. To see exactly how they transform notice that, according to (11) and (13) the two neurons form a (mutually dependent) triplet $\left(A_{\mu}^{1}, A_{\mu}^{2}, A_{\mu}^{3}\right)$ and the six chromons form a sextet $\left(\vec{R}_{\mu}, \vec{B}_{\mu}, \vec{G}_{\mu}, \vec{R}_{\mu}^{(c)}, \vec{B}_{\mu}^{(c)}, \vec{G}_{\mu}^{(c)}\right)$ or equivalently $\left(R_{\mu}, B_{\mu}, G_{\mu}, \bar{R}_{\mu}, \bar{B}_{\mu}, \bar{G}_{\mu}\right)$. For the neurons we have

$$
\begin{aligned}
& R_{2}:\left(\begin{array}{l}
A_{\mu} \\
A_{\mu}^{\prime}
\end{array}\right) \rightarrow\left(\begin{array}{cc}
-1 & 0 \\
0 & 1
\end{array}\right)\left(\begin{array}{l}
A_{\mu} \\
A_{\mu}^{\prime}
\end{array}\right), \\
& R_{3}:\left(\begin{array}{c}
A_{\mu} \\
A_{\mu}^{\prime}
\end{array}\right) \rightarrow\left(\begin{array}{cc}
1 / 2 & \sqrt{3} / 2 \\
\sqrt{3} / 2 & -1 / 2
\end{array}\right)\left(\begin{array}{c}
A_{\mu} \\
A_{\mu}^{\prime}
\end{array}\right),
\end{aligned}
$$

from which we have

$$
\begin{aligned}
& R_{2}:\left(A_{\mu}^{1}, A_{\mu}^{2}, A_{\mu}^{3}\right) \rightarrow-\left(A_{\mu}^{1}, A_{\mu}^{3}, A_{\mu}^{2}\right), \\
& R_{3}:\left(A_{\mu}^{1}, A_{\mu}^{2}, A_{\mu}^{3}\right) \rightarrow-\left(A_{\mu}^{3}, A_{\mu}^{2}, A_{\mu}^{1}\right), \\
& R_{4}:\left(A_{\mu}^{1}, A_{\mu}^{2}, A_{\mu}^{3}\right) \rightarrow-\left(A_{\mu}^{2}, A_{\mu}^{1}, A_{\mu}^{3}\right), \\
& R_{5}:\left(A_{\mu}^{1}, A_{\mu}^{2}, A_{\mu}^{3}\right) \rightarrow\left(A_{\mu}^{3}, A_{\mu}^{1}, A_{\mu}^{2}\right), \\
& R_{6}:\left(A_{\mu}^{1}, A_{\mu}^{2}, A_{\mu}^{3}\right) \rightarrow\left(A_{\mu}^{2}, A_{\mu}^{3}, A_{\mu}^{1}\right) .
\end{aligned}
$$

This tells that basically $R_{2}, R_{3}, R_{4}$ represent the permutations of two SU(2) neurons (up to the signature change), but $R_{5}$, $R_{6}$ represent the cyclic permutations of three $\mathrm{SU}(2)$ neurons.

For the chromons the color reflection acts as follows,

$$
\begin{gathered}
R_{2}:\left(R_{\mu}, B_{\mu}, G_{\mu}, \bar{R}_{\mu}, \bar{B}_{\mu}, \bar{G}_{\mu}\right) \\
\longrightarrow\left(\bar{R}_{\mu}, \bar{G}_{\mu}, \bar{B}_{\mu}, R_{\mu}, G_{\mu}, B_{\mu}\right), \\
R_{3}:\left(R_{\mu}, B_{\mu}, G_{\mu}, \bar{R}_{\mu}, \bar{B}_{\mu}, \bar{G}_{\mu}\right) \\
\longrightarrow-\left(\bar{G}_{\mu}, \bar{B}_{\mu}, \bar{R}_{\mu}, G_{\mu}, B_{\mu}, R_{\mu}\right), \\
\quad \longrightarrow \quad\left(R_{\mu}, B_{\mu}, G_{\mu}, \bar{R}_{\mu}, \bar{B}_{\mu}, \bar{G}_{\mu}\right) \\
\left.R_{4}\right) \\
\longrightarrow-\left(\bar{B}_{\mu}, \bar{R}_{\mu}, \bar{G}_{\mu}, B_{\mu}, R_{\mu}, G_{\mu}\right), \\
R_{5}:\left(R_{\mu}, B_{\mu}, G_{\mu}, \bar{R}_{\mu}, \bar{B}_{\mu}, \bar{G}_{\mu}\right) \\
\longrightarrow-\left(G_{\mu}, R_{\mu}, B_{\mu}, \bar{G}_{\mu}, \bar{R}_{\mu}, \bar{B}_{\mu}\right), \\
R_{6}:\left(R_{\mu}, B_{\mu}, G_{\mu}, \bar{R}_{\mu}, \bar{B}_{\mu}, \bar{G}_{\mu}\right) \\
\longrightarrow-\left(B_{\mu}, G_{\mu}, R_{\mu}, \bar{B}_{\mu}, \bar{G}_{\mu}, \bar{R}_{\mu}\right) .
\end{gathered}
$$

Here $R_{2}, R_{3}, R_{4}$ denote the anti-chromon transformation (complex conjugation) plus permutations of two chromons, but $R_{5}, R_{6}$ denote the cyclic permutations of three chromons (up to the signature change). Just as in SU(2) QCD here the complex conjugation (anti-chromon transformation) of the chromons in the color reflection plays the crucial role in the calculation of the effective action.

The above analysis reveals another important difference between the neuron and chromon. Clearly (28) tells that the neurons permute amomg themselves, but (29) tells that the chromons transform to anti-chromons, under the color reflection. In other words, just like the photon in QED the neurons have no anti-neurons. In comparison the chromons have the anti-chromon partners. This is because the neurons are color neutral so that they have the real representation, while the chromons are colored and allow the complex representation.

At this point one might wonder if there is any relation between the color reflection group and Weyl group. For SU(3), the Weyl group is the six elements permutation group of three colors which has a three-dimensional representation given by

$$
\begin{array}{ll}
W_{1}=\left(\begin{array}{lll}
1 & 0 & 0 \\
0 & 1 & 0 \\
0 & 0 & 1
\end{array}\right), & W_{2}=\left(\begin{array}{lll}
0 & 1 & 0 \\
1 & 0 & 0 \\
0 & 0 & 1
\end{array}\right), \\
W_{3}=\left(\begin{array}{lll}
1 & 0 & 0 \\
0 & 0 & 1 \\
0 & 1 & 0
\end{array}\right), & W_{4}=\left(\begin{array}{lll}
0 & 0 & 1 \\
0 & 1 & 0 \\
1 & 0 & 0
\end{array}\right), \\
W_{5}=\left(\begin{array}{lll}
0 & 1 & 0 \\
0 & 0 & 1 \\
1 & 0 & 0
\end{array}\right), & W_{6}=\left(\begin{array}{lll}
0 & 0 & 1 \\
1 & 0 & 0 \\
0 & 1 & 0
\end{array}\right),
\end{array}
$$

which contains the cyclic $Z_{3}$ made of $W_{1}, W_{5}$, and $W_{6}$. 
This tells that the two groups are different. They have different origin. The Weyl group comes as the symmetry of the Abelian decomposition, but the color reflection group comes as the residual symmetry of the Abelian decomposition. Unlike the color reflection group (26), the Weyl group (30) is not a subgroup of SU(3). Moreover, the Weyl group has no complex conjugation operation which transforms the chromons to anti-chromons. On the other hand they have a common subgroup $Z_{3}$, the cyclic permutation group of three colors.

Both the color reflection group and the Weyl group play a fundamental role in hadron spectroscopy. Only the color reflection invariant and Weyl invariant combinations of quarks and gluons can become physical in the quark and chromon model [47]. Moreover, they play the crucial role for us to calculate the one-loop effective action of SU(3) QCD and prove the monopole condensation gauge invariantly.

\section{One-loop effective action of SU(2) QCD: a review}

Before we calculate the one-loop effective action of SU(3) QCD we need to understand how we calculate the effective action of the SU(2) QCD, for two reasons. First, the calculation of the SU(2) QCD effective action becomes an essential part for the calculation of the effective action of SU(3) QCD. Second, the early calculations had critical defects [17-26]. The Savvidy vacuum was unstable. More seriously, it was not gauge invariant. So we have to know how to correct these critical mistakes.

To obtain the one-loop effective action we must divide the gauge potential to the classical and quantum parts and integrate out the quantum part in the presence of the classical background. The Abelian decomposition naturally provides an ideal platform for this, since we can treat the Abelian part as the classical background and integrate out the valence part.

Imposing the quantum gauge fixing condition $\bar{D}_{\mu} \vec{X}_{\mu}=0$ we have [30-33],

$$
\begin{aligned}
& \exp \left[i S_{\text {eff }}\left(\hat{A}_{\mu}\right)\right]=\int \mathcal{D} \vec{X}_{\mu} \mathcal{D} \vec{X}_{\mu}^{(c)} \mathcal{D} \vec{c} \mathcal{D} \vec{c}^{*} \\
& \exp \left\{i \int \left[-\frac{1}{4} \hat{F}_{\mu \nu}^{2}-\frac{1}{4}\left(\hat{D}_{\mu} \vec{X}_{\nu}-\hat{D}_{\nu} \vec{X}_{\mu}\right)^{2}\right.\right. \\
& -\frac{g}{2} \hat{F}_{\mu \nu} \cdot\left(\vec{X}_{\mu} \times \vec{X}_{\nu}\right)-\frac{g^{2}}{4}\left(\vec{X}_{\mu} \times \vec{X}_{\nu}\right)^{2} \\
& \left.\left.+\vec{c}^{*} \bar{D}_{\mu} D_{\mu} \vec{c}-\frac{1}{2 \xi}\left(\bar{D}_{\mu} \vec{X}_{\mu}\right)^{2}\right] d^{4} x\right\},
\end{aligned}
$$

where $\vec{c}$ and $\vec{c}^{*}$ are the ghost fields. But notice that the quartic interaction of $\vec{X}_{\mu}$ can be neglected since this does not contribute in the one-loop integration.

The above integral expression of the effective action has the following advantages. First, the separation of the classical and quantum parts is explicitly gauge independent. Second, the functional integration of is made by the chromon and anti-chromon in the color reflection symmetric way. As we will see, this point plays the crucial role for us to implement the gauge invariance in the functional integral.

Clearly these salient features were lacking in the old calculations [17-26]. The classical part was not Abelian, and the separation of the classical and quantum parts was ad hoc. Moreover, all three gluons were integrated in the functional integration. In particular, the role of the anti-chromon in the functional integral was completely obscure, which has made the implementation of the gauge invariance very difficult in the functional integral. This was because the Abelian decomposition was not available at that time.

A more serious problem of the old calculations, however, was the wrong background. The Abelian decomposition tells that there are two possible backgrounds, the non-topological $F_{\mu \nu}$ and the topological $H_{\mu \nu}$. But in old calculations people have chosen $F_{\mu \nu}$ which is not gauge invariant nor parity conserving. Moreover, this does not describe the monopole background. This is the problem with the Savvidy vacuum [17-26].

Let us choose the wrong background for the moment, and let

$\bar{F}_{\mu \nu}=H \delta_{[\mu}^{1} \delta_{\nu]}^{2}$.

where $H$ is a constant magnetic field of $F_{\mu \nu}$ in $z$-direction. With this the effective action is expressed by the chromon and ghost loop determinants given by $K$ and $M$ [17-26],

$$
\begin{aligned}
\Delta S & =\frac{i}{2} \ln \operatorname{Det} K-i \ln \operatorname{Det} M, \\
\operatorname{Det}^{-1 / 2} K_{\mu \nu} & =\operatorname{Det}\left(-g_{\mu \nu} \bar{D}^{2}+2 i g \bar{F}_{\mu \nu}\right), \\
\operatorname{Det} M^{1 / 2} & =\operatorname{Det}\left(-\bar{D}^{2}\right),
\end{aligned}
$$

where $\bar{D}_{\mu}=\partial_{\mu}-g \bar{A}_{\mu} \hat{n} \times$ is the covariant derivative defined by the classical background.

One can calculate the functional determinant of the gluon loop from the energy spectrum of a massless charged vector field moving around the constant magnetic field $H$, which is given by [52]

$E^{2}=2 g H\left(n+\frac{1}{2}-q S_{3}\right)+k^{2}$,

where $S_{3}$ and $k$ are the spin and momentum of the vector field in the direction of the magnetic field, and $q= \pm 1$ is the charge of the vector field. Notice that the energy spectrum of gluons for two different spin polarizations $S_{3}= \pm 1$ is different. Moreover, when $S_{3}=1$, it contains negative (tachyonic) eigenvalues which violate the causality. This is schematically shown in Fig. 6 for $q=+1$ in (a) and $q=-1$ in (b). 


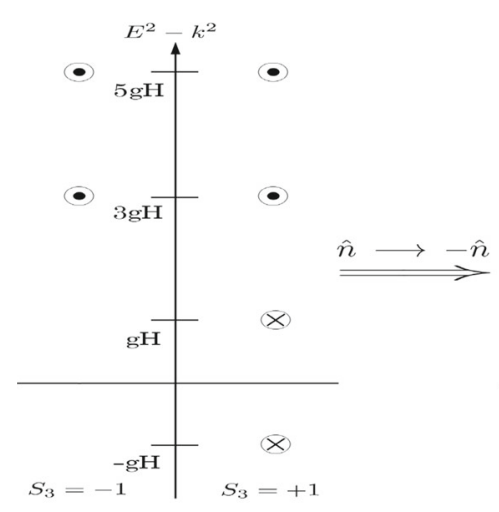

(A)

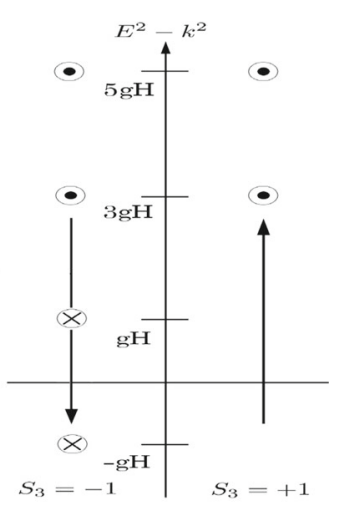

(B)

Fig. 6 The gauge invariant eigenvalues of the gluon functional determinant. Notice that the color reflection invariance removes the tachyonic modes in both $\mathbf{a}$ and $\mathbf{b}$

From this one has the integral expression of the effective action given by [17-26]

$$
\begin{aligned}
\Delta S= & i \ln \operatorname{Det}\left[\left(-\bar{D}^{2}+g H\right)\left(-\bar{D}^{2}-g H\right)\right] \\
& -2 i \ln \operatorname{Det}\left(-\bar{D}^{2}\right), \\
\Delta \mathcal{L}= & \lim _{\epsilon \rightarrow 0} \frac{\mu^{2}}{16 \pi^{2}} \int_{0}^{\infty} \frac{d t}{t^{2-\epsilon}} \frac{g H}{\sinh \left(g H t / \mu^{2}\right)} \\
& \times\left[\exp \left(-2 g H t / \mu^{2}\right)+\exp \left(+2 g H t / \mu^{2}\right)\right] .
\end{aligned}
$$

Notice that the two exponential terms which represent the contribution of two spin polarizations is different. Moreover, the second term has a severe infra-red divergence.

With the standard $\zeta$-function regularization one can integrate (35) and obtain the SNO effective action [17-23]

$$
\begin{aligned}
\mathcal{L}_{e f f}= & -\frac{1}{2} H^{2}-\frac{11 g^{2}}{48 \pi^{2}} H^{2}\left(\ln \frac{g H}{\mu^{2}}-c\right) \\
& +i \frac{g^{2}}{8 \pi} H^{2},
\end{aligned}
$$

where $c$ is an integration constant. This contains the wellknown imaginary part coming from the tachyonic eigenstates, which destablizes the Savvidy vacuum [18-26].

There have been huge efforts to cure this instability of the Savvidy vacuum [18-26]. Actually there are ways to cure the instability. One way is to impose the causality in the functional integral $[30,31]$. Clearly the causality removes the tachyonic modes, and remove the imaginary part. Another is to calculate the imaginary part perturbatively to the second order in the coupling constant $g[32,33]$. This is because the imaginary part at one loop level is in the order $g^{2}$, although in principle the effective action is non-perturbative. And the perturbative calculation confirms that there should be no imaginary part.

But we emphasize that this instability is not the only problem of the Savvidy vacuum. There are other problems. For

instance, it does not describe the monopole condensation. But the most serious problem is that it is not gauge invariant nor parity conserving, and thus can not be identified as the QCD vacuum.

Another serious defect in the above calculation is that the gauge invariance is not correctly implemented in the calculation. We could implement the gauge invariance in the old calculations, but there is no point to do so. First of all, this does not change the result. More seriously, it is meaningless and irrelevant to do so because the gauge invariance has already been compromised as soon as the non-topological background (32) was chosen.

Fortunately the Abelian decomposition allows us to calculate the effective action correctly [30-34]. First, it allows us to separate the gauge invariant and parity conserving monopole background gauge independently. Second, it allows us to impose the much simpler color reflection invariance to implement the gauge invariance in the calculation of the effective action. This makes the calculation simple and clear.

To show this we first make the Abelian decomposition and choose the gauge invariant and parity conserving monopole background $H_{\mu \nu}[34,46]$

$\bar{H}_{\mu \nu}=\bar{H} \delta_{[\mu}^{1} \delta_{v]}^{2}$,

where $\bar{H}$ now is a constant chromo-magnetic field of $H_{\mu \nu}$ in $z$-direction. With this we can integrate out the chromon and express the effective action by the chromon and ghost loop determinants [30-34],

$$
\begin{aligned}
\Delta S & =\frac{i}{2} \ln \operatorname{Det} K-i \ln \operatorname{Det} M, \\
\operatorname{Det}^{-1 / 2} K_{\mu \nu} & =\operatorname{Det}\left(-g_{\mu \nu} \bar{D}^{2}+2 i g \bar{H}_{\mu \nu}\right), \\
\operatorname{Det} M^{1 / 2} & =\operatorname{Det}\left(-\bar{D}^{2}\right),
\end{aligned}
$$

where $\bar{D}_{\mu}$ is the covariant derivative defined by the monopole background.

The next step is to calculate the chromon loop functional determinant implementing the gauge invariance. Clearly the energy spectrum of the chromon moving around the constant magnetic field $\bar{H}$ is given by (34) as before, but here we have to find the gauge invariant energy spectrum. A simplest way to do that is to choose the energy eigenvalues which remain invariant under the color reflection. This is because the color reflection invariance is synonymous to the gauge invariance after the Abelian decomposition.

Under the color reflection the chromon undergoes to the complex conjugation and becomes anti-chromon which has opposite color charge. So the energy spectrum shown in Fig. 6 a for $q=+1$ changes to (b) for $q=-1$. As we have emphasized, however, physics should not change under this color reflection. In particular, the eigenvalues of the chromon functional determinant for each spin polarization should remain the same. 


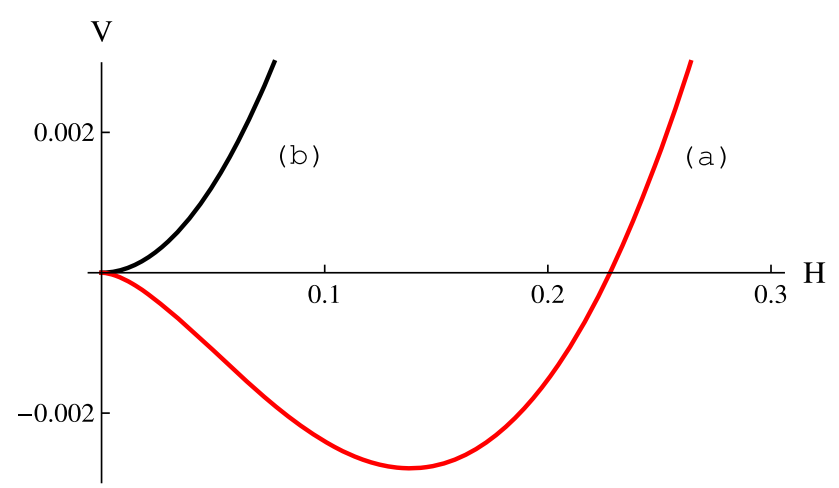

Fig. 7 The effective potential of SU(2) QCD in the pure magnetic background. Here (a) is the effective potential and (b) is the classical potential

This means that only the eigenvalues which appear in both (a) and (b) simultaneously become gauge invariant and physical. This excludes the lowest two (in particular the tachyonic) eigenvalues in both (a) and (b). This is the C-projection which removes the tachyonic modes and makes the monopole condensation stable [34].

This tells that the color reflection invariance plays exactly the same role as the G-parity in string theory. In the NSR string theory the GSO projection restores the supersymmetry and modular invariance by projecting out the tachyonic vacuum [27-29]. Just like the GSO-projection in string theory, the C-projection in QCD removes the tachyonic modes and restores the gauge invariance which assures the stable monopole condensation.

So, imposing the color reflection invariance we have [3034],

$$
\begin{aligned}
\Delta S= & i \ln \operatorname{Det}\left[\left(-\bar{D}^{2}+g \bar{H}\right)\left(-\bar{D}^{2}+g \bar{H}\right)\right] \\
& -2 i \ln \operatorname{Det}\left(-\bar{D}^{2}\right), \\
\Delta \mathcal{L}= & \lim _{\epsilon \rightarrow 0} \frac{\mu^{2}}{16 \pi^{2}} \int_{0}^{\infty} \frac{d t}{t^{2-\epsilon}} \frac{g \bar{H}}{\sinh \left(g \bar{H} t / \mu^{2}\right)} \\
& \times\left[\exp \left(-2 g \bar{H} t / \mu^{2}\right)+\exp \left(-2 g \bar{H} t / \mu^{2}\right)\right] .
\end{aligned}
$$

This should be compared with (35). Here the two chromon spin polarization contributions shown in the two exponential terms become identical. Moreover, we have no infra-red divergence here. This, of course, is because the color reflection invariance removes the eigenvalues which are not gauge invariant. Integrating this we have the SU(2) QCD effective action

$$
\mathcal{L}_{\text {eff }}=-\frac{1}{2} \bar{H}^{2}-\frac{11 g^{2}}{48 \pi^{2}} \bar{H}^{2}\left(\ln \frac{g \bar{H}}{\mu^{2}}-c\right)
$$

Clearly the effective action has no imaginary part at all.

The effective action (40) generates the much desired dimensional transmutation in QCD. From (40) we have the following effective potential
$V=\frac{1}{2} \bar{H}^{2}\left[1+\frac{11 g^{2}}{24 \pi^{2}}\left(\ln \frac{g \bar{H}}{\mu^{2}}-c\right)\right]$.

With this we define the running coupling $\bar{g}$ by [30-33]

$$
\begin{aligned}
\left.\frac{\partial^{2} V}{\partial \bar{H}^{2}}\right|_{\bar{H}=\bar{\mu}^{2} / g} & =\frac{g^{2}}{\bar{g}^{2}} \\
& =1+\frac{11 g^{2}}{24 \pi^{2}}\left(\ln \frac{\bar{\mu}^{2}}{\mu^{2}}-c+\frac{3}{2}\right),
\end{aligned}
$$

and retrieve the well known $\beta$-function which explains the asymptotic freedom $[53,54]$

$\beta(\bar{\mu})=\bar{\mu} \frac{\partial \bar{g}}{\partial \bar{\mu}}=-\frac{11 \bar{g}^{3}}{24 \pi^{2}}$.

This confirms that our calculation is consistent with the known result.

In terms of the running coupling the renormalized potential is given by

$V_{\text {ren }}=\frac{1}{2} \bar{H}^{2}\left[1+\frac{11 \bar{g}^{2}}{24 \pi^{2}}\left(\ln \frac{\bar{g} \bar{H}}{\bar{\mu}^{2}}-\frac{3}{2}\right)\right]$,

which generates a non-trivial local minimum at

$\langle\bar{H}\rangle=\frac{\bar{\mu}^{2}}{\bar{g}} \exp \left(-\frac{24 \pi^{2}}{11 \bar{g}^{2}}+1\right)$.

The corresponding effective potential is plotted in Fig. 7 where we have assumed $\bar{\alpha}_{s}=1$ and $\bar{\mu}=1$. This is nothing but the monopole condensation which generates the desired mass gap and dimensional transmutation in QCD.

To summarize, the gauge invariant and parity conserving monopole background and the color reflection invariance allows us to demonstrate the stable monopole condensation and the generation of the mass gap in SU(2) QCD gauge independently. In particular, the color reflection invariance (the C-projection) removes the tachyonic modes which destabilized the Savvidy vacuum.

Notice that the monopole background could also contain the electric (i.e., Coulomb) part. And we can generalise the above calculation with an arbitrary chromo-electromagnetic monopole background. To do so we choose an arbitrary $\bar{H}_{\mu \nu}$ which has constant electric and magnetic fields $\bar{E}$ and $\bar{H}$ given by

$$
\begin{aligned}
& \bar{H}=\frac{1}{2} \sqrt{\sqrt{\bar{H}_{\mu \nu}^{4}+\left(\bar{H}_{\mu \nu} \tilde{\bar{H}}_{\mu \nu}\right)^{2}}+\bar{H}_{\mu \nu}^{2},} \\
& \bar{E}=\frac{1}{2} \sqrt{\sqrt{\bar{H}_{\mu \nu}^{4}+\left(\bar{H}_{\mu \nu} \tilde{\bar{H}}_{\mu \nu}\right)^{2}}-\bar{H}_{\mu \nu}^{2}} .
\end{aligned}
$$

With this we can integrate out the chromon pair gauge invariantly, imposing the color reflection invariance.

Since the color reflection invariance assures that the two spin polarizations of the chromon have exactly the same contribution in the effective action, we have [30-34]

$$
\Delta S=i \ln \operatorname{Det}\left[\left(-\bar{D}^{2}+g \bar{H}\right)\left(-\bar{D}^{2}+g \bar{H}\right)\right]
$$




$$
\begin{aligned}
& +i \ln \operatorname{Det}\left[\left(-\bar{D}^{2}-i g \bar{E}\right)\left(-\bar{D}^{2}-i g \bar{E}\right)\right] \\
& -2 i \ln \operatorname{Det}\left(-\bar{D}^{2}\right), \\
\Delta \mathcal{L}= & \lim _{\epsilon \rightarrow 0} \frac{g^{2}}{8 \pi^{2}} \int_{0}^{\infty} \frac{d t}{t^{1-\epsilon}} \frac{\bar{H} \bar{E}}{\sinh \left(g \bar{H} t / \mu^{2}\right) \sin \left(g \bar{E} t / \mu^{2}\right)} \\
& \times\left[\exp \left(-2 g \bar{H} t / \mu^{2}\right)+\exp \left(+2 i g \bar{E} t / \mu^{2}\right)-1\right] .
\end{aligned}
$$

This is the correct integral expression of SU(2) QCD effective action.

Integrating this we have

$$
\mathcal{L}_{e f f}=\left\{\begin{array}{l}
-\frac{1}{2} \bar{H}^{2}-\frac{11 g^{2}}{48 \pi^{2}} \bar{H}^{2}\left(\ln \frac{g \bar{H}}{\mu^{2}}-c^{\prime}\right), \quad \bar{E}=0 \\
\frac{1}{2} g^{2} \bar{E}^{2}+\frac{11 g^{2}}{48 \pi^{2}} \bar{E}^{2}\left(\ln \frac{g \bar{E}}{\mu^{2}}-c^{\prime}\right) \\
-i \frac{11 g^{2}}{96 \pi} \bar{E}^{2}, \quad \bar{H}=0
\end{array}\right.
$$

Notice that when $\bar{H}=0$ it has a negative imaginary part, which implies the pair annihilation of chromons [30-33,55]. This must be contrasted with the QED effective action where the electron loop generates a positive imaginary part [5659]. This difference is a direct consequence of the BoseEinstein statistics of the chromon loop. Of course the quark loop in QCD, due to the Fermi-Dirac statistics, will generate a positive imaginary part which diminishes the asymptotic freedom [30-33].

This has a deep meaning. The positive imaginary part in QED means the pair creation which generates the screening. On the other hand in QCD we must have the anti-screening to explain the asymptotic freedom, and the negative imaginary part is exactly what we need for the asymptotic freedom [30$33,55]$.

The effective action (48) has an important symmetry, the electric-magnetic duality $[30,31]$. We can obtain the two effective actions for $\bar{H}=0$ and $\bar{E}=0$ from each other simply with the following replacement

$\bar{E} \rightarrow i \bar{H}, \quad \bar{H} \rightarrow-i \bar{E}$.

This duality was first discovered in the QED effective action [56-58]. But subsequently this duality has been shown to exist also in the QCD effective action [30-33].

This tells that the duality should be regarded as a fundamental symmetry of the effective action of gauge theory, Abelian and non-Abelian. The importance of this duality is that it provides a very useful tool to check the self-consistency of the effective action. The fact that the two effective actions are related by the duality assures that they are self-consistent. Notice that this duality is different from the well known duality in Maxwell's theory, that the theory is invariant under $\bar{E} \rightarrow \bar{H}, \bar{H} \rightarrow-\bar{E}$.

\section{One-loop effective action of SU(3) QCD and monopole condensation}

To obtain the one-loop SU(3) effective action we follow the same procedure. Make the Abelian decomposition and integrate out the colored valence gluons $\vec{X}_{\mu}$ (three chromons $\vec{W}_{\mu}^{p}$ ) gauge invariantly with the monopole field as the classical background, imposing the color reflection invariance. What is really remarkable is that the Weyl symmetry of the Abelian decomposition greatly simplifies this procedure.

From the Weyl symmetric Lagrangian (16) we have

$$
\begin{aligned}
& \exp \left[i S_{e f f}\left(\hat{A}_{\mu}\right)\right]=\int \Pi_{p} \mathcal{D} \vec{W}_{\mu}^{p} \mathcal{D} \vec{c}^{p} \mathcal{D} \vec{c}^{p *} \\
& \exp \left\{i \int \left[\sum _ { p } \left\{-\frac{1}{6}\left(\hat{F}_{\mu \nu}^{p}\right)^{2}-\frac{1}{4}\left(\hat{D}_{\mu}^{p} \vec{W}_{\nu}^{p}-\hat{D}_{\nu}^{p} \vec{W}_{\mu}^{p}\right)^{2}\right.\right.\right. \\
& \left.-\frac{g}{2} \hat{F}_{\mu \nu}^{p} \cdot\left(\vec{W}_{\mu}^{p} \times \vec{W}_{\nu}^{p}\right)\right\}-\sum_{p, q} \frac{g^{2}}{4}\left(\vec{W}_{\mu}^{p} \times \vec{W}_{\mu}^{q}\right)^{2} \\
& -\sum_{p, q, r} \frac{g}{2}\left(\hat{D}_{\mu}^{p} \vec{W}_{\nu}^{p}-\hat{D}_{\nu}^{p} \vec{W}_{\mu}^{p}\right) \cdot\left(\vec{W}_{\mu}^{q} \times \vec{W}_{\mu}^{r}\right) \\
& -\sum_{p \neq q} \frac{g^{2}}{4}\left(\left(\vec{W}_{\mu}^{p} \times \vec{W}_{\nu}^{q}\right) \cdot\left(\vec{W}_{\mu}^{q} \times \vec{W}_{\nu}^{p}\right)\right. \\
& \left.+\left(\vec{W}_{\mu}^{p} \times \vec{W}_{\nu}^{p}\right) \cdot\left(\vec{W}_{\mu}^{q} \times \vec{W}_{\nu}^{q}\right)\right) \\
& \left.\left.+\sum_{p} \vec{c}^{p *} \bar{D}_{\mu} D_{\mu} \vec{c}^{p}-\frac{1}{2 \xi} \sum_{p}\left(\bar{D}_{\mu}^{p} \vec{W}_{\mu}^{p}\right)^{2}\right] d^{4} x\right\}
\end{aligned}
$$

where we have imposed the gauge fixing condition $\hat{D}_{\mu} \vec{X}_{\mu}=$ $\sum_{p}\left(\bar{D}_{\mu}^{p} \vec{W}_{\mu}^{p}\right)=0$.

At the first glance the integral looks complicated, but there are two things which simplify the integral. First, in the one loop approximation only the terms quadratic in $\vec{W}_{\mu}^{p}$ contribute to the integral. Second, the Weyl symmetric Abelian decomposition (16) reduces the chromon functional integral to the sum of three $\mathrm{SU}(2)$ integral of $\vec{W}_{\mu}^{p}$.

So the integral expression of the effective action is simplified to

$$
\begin{aligned}
& \exp \left[i S_{\text {eff }}\left(\hat{A}_{\mu}\right)\right] \simeq \sum_{p} \int \mathcal{D} \vec{W}_{\mu}^{p} \mathcal{D} \vec{c}^{p} \mathcal{D} \vec{c}^{p *} \\
& \quad \exp \left\{i \int \left[-\frac{1}{6}\left(\hat{F}_{\mu \nu}^{p}\right)^{2}-\frac{1}{4}\left(\hat{D}_{\mu}^{p} \vec{W}_{\nu}^{p}-\hat{D}_{\nu}^{p} \vec{W}_{\mu}^{p}\right)^{2}\right.\right. \\
& \quad-\frac{g}{2} \hat{F}_{\mu \nu}^{p} \cdot\left(\vec{W}_{\mu}^{p} \times \vec{W}_{\nu}^{p}\right) \\
& \left.\left.\quad+\vec{c}^{p *} \bar{D}_{\mu} D_{\mu} \vec{c}^{p}-\frac{1}{2 \xi}\left(\bar{D}_{\mu}^{p} \vec{W}_{\mu}^{p}\right)^{2}\right] d^{4} x\right\}
\end{aligned}
$$

This effectively reduces the calculation of the SU(3) QCD effective action to that of SU(2) QCD calculation. In general this applies to the $\mathrm{SU}(\mathrm{N})$ QCD effective action, because the Weyl symmetry holds in the Abelian decomposition of any 
SU(N) QCD. This is why the calculation of the SU(2) QCD effective action is so important. This simplification would have been impossible without the Weyl symmetric Abelian decomposition.

Now, all we have to do is to add the SU(2) result (48) in a Weyl symmetric way. With the constant monopole background $\overline{\bar{H}}_{\mu \nu}^{p}$ given by

$$
\begin{aligned}
& \bar{H}_{p}=\frac{1}{2} \sqrt{\sqrt{\left(\bar{H}_{\mu \nu}^{p}\right)^{4}+\left(\bar{H}_{\mu \nu}^{p} \tilde{\bar{H}}_{\mu \nu}^{p}\right)^{2}}+\left(\bar{H}_{\mu \nu}^{p}\right)^{2}}, \\
& \bar{E}_{p}=\frac{1}{2} \sqrt{\sqrt{\left(\bar{H}_{\mu \nu}^{p}\right)^{4}+\left(\bar{H}_{\mu \nu}^{p} \tilde{\bar{H}}_{\mu \nu}^{p}\right)^{2}}-\left(\bar{H}_{\mu \nu}^{p}\right)^{2}},
\end{aligned}
$$

we have

$$
\begin{aligned}
\Delta S= & 2 i \sum_{p} \ln \operatorname{Det}\left[\left(-\bar{D}_{p}^{2}+2 g \bar{H}_{p}\right)\left(-\bar{D}_{p}^{2}-2 i g \bar{E}_{p}\right)\right] \\
& -2 i \sum_{p} \ln \operatorname{Det}\left(-\bar{D}_{p}^{2}\right)
\end{aligned}
$$

so that

$$
\begin{aligned}
\Delta \mathcal{L}= & \lim _{\epsilon \rightarrow 0} \sum_{p} \frac{g^{2}}{8 \pi^{2}} \int_{0}^{\infty} \frac{d t}{t^{1-\epsilon}} \frac{\bar{H}_{p} \bar{E}_{p}}{\sinh \left(g \bar{H}_{p} t / \mu^{2}\right) \sin \left(g \bar{E}_{p} t / \mu^{2}\right)} \\
& \times\left[\exp \left(-2 g \bar{H}_{p} t / \mu^{2}\right)+\exp \left(+2 i g \bar{E}_{p} t / \mu^{2}\right)-1\right] .
\end{aligned}
$$

Notice that for the chromo-magnetic background we have $\bar{E}_{p}=0$, but for the chromo-electric background we have $\bar{H}_{p}=0$.

From this we have the following explicitly Weyl symmetric effective Lagrangian. For $\bar{E}_{p}=0$ we have

$$
\mathcal{L}_{e f f}=-\sum_{p}\left(\frac{\bar{H}_{p}^{2}}{3}+\frac{11 g^{2}}{48 \pi^{2}} \bar{H}_{p}^{2}\left(\ln \frac{g \bar{H}_{p}}{\mu^{2}}-c\right)\right),
$$

and for $\bar{H}_{p}=0$ we have

$$
\begin{aligned}
\mathcal{L}_{e f f}= & \sum_{p}\left(\frac{\bar{E}_{p}^{2}}{3}+\frac{11 g^{2}}{48 \pi^{2}} \bar{E}_{p}^{2}\left(\ln \frac{g \bar{E}_{p}}{\mu^{2}}-c\right)\right. \\
& \left.-i \frac{11 g^{2}}{96 \pi} \bar{E}_{p}^{2}\right) .
\end{aligned}
$$

Just as in $\mathrm{SU}(2)$, the effective action has a negative imaginary part when $\bar{H}_{p}=0$. This again tells that the chromo-electric field annihilates the chromon pairs, which implies the antiscreening and asymptotic freedom [30-34,53,54]. Moreover, the effective action has the dual symmetry. It is invariant under the dual transformation $\bar{H}_{p} \rightarrow-i \bar{E}_{p}$ and $\bar{E}_{p} \rightarrow i \bar{H}_{p}$.

We can express the effective Lagrangians (55) and (56) in terms of three Casimir invariants $C_{p}$ of SU(3). For example, for the pure chromo-magnetic background $\bar{H}_{\mu \nu}^{a}=\bar{H}_{\mu \nu} n^{a}+$ $\bar{H}_{\mu \nu}^{\prime} n^{\prime a}$ we have

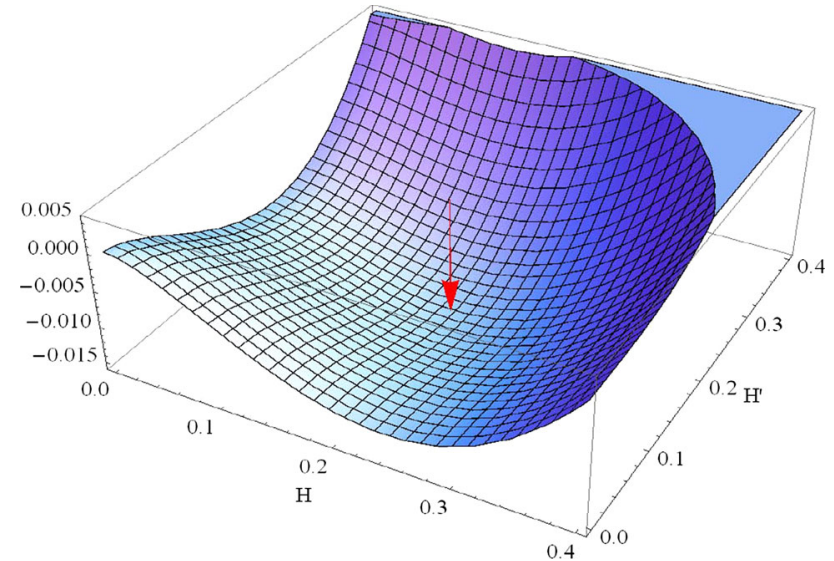

Fig. 8 The QCD effective potential with $\cos \theta=0$, which has a unique minimum at $H=H^{\prime}=H_{0}$

$$
\begin{aligned}
& C_{1}=\left(\bar{H}_{\mu \nu}^{a}\right)^{2}, \\
& C_{2}=\left(d^{a b c} \bar{H}_{\mu \nu}^{b} \bar{H}_{\mu \nu}^{c}\right)^{2}, \\
& C_{3}=\left(d^{a b c} \bar{H}_{\mu \nu}^{a} \bar{H}_{\nu \rho}^{b} \bar{H}_{\rho \mu}^{c}\right)^{2},
\end{aligned}
$$

which are related to $\bar{H}_{p}$ by the following identities

$$
\begin{aligned}
& \bar{H}_{1}^{2}+\bar{H}_{2}^{2}+\bar{H}_{3}^{2}=\frac{3}{2} C_{1}, \\
& \bar{H}_{1} \bar{H}_{2}+\bar{H}_{2} \bar{H}_{3}+\bar{H}_{3} \bar{H}_{1}=\frac{3}{4} C_{1}^{2}-\frac{9}{16} C_{2}, \\
& \bar{H}_{1} \bar{H}_{2} \bar{H}_{3}=\frac{1}{8} C_{1}^{3}-\frac{3}{16} C_{1} C_{2}-\frac{3}{2} C_{3} .
\end{aligned}
$$

So, expressing $\bar{H}_{p}$ by $C_{p}$ we can replace $\bar{H}_{p}$ in (55) by $C_{p}$. Similarly, for the pure chromo-electric (Coulombic) background we have exactly the same identities, with $\bar{H}_{p}$ replaced by $\bar{E}_{p}$. This assures that we can express the effective Lagrangian by three Casimir invariants.

Notice that the classical potential depends on only one Casimir invariant $C_{1}=\bar{H}_{1}^{2}+\bar{H}_{2}^{2}+\bar{H}_{3}^{2}$, but the effective potential depends on all three Carsimir invariants (or equivalently three independent variables $\bar{H}_{1}, \bar{H}_{2}$, and $\bar{H}_{3}$ ). On the other hand, the chromo-magnetic background in SU(3) QCD is given by seemingly two independent monopole fields $\vec{H}$ and $\vec{H}^{\prime}$. So we need to understand what is the origin of the third degree.

To understand this notice that $\vec{H}$ and $\vec{H}^{\prime}$ in principle can have different space orientation, so that the angle $\theta$ which describes the relative orientation of two vectors $\vec{H}$ and $\vec{H}^{\prime}$ in real space can be arbitrary. So the constant chromo-magnetic background has three degrees, $\bar{H}, \bar{H}^{\prime}$ and $\theta$. In fact from (11) we have

$$
\begin{aligned}
\bar{H}_{1} & =|\vec{H}|, \quad \bar{H}_{2}=\bar{H}_{+}, \quad \bar{H}_{3}=\bar{H}_{-}, \\
H_{ \pm} & =\left|\frac{1}{2} \vec{H} \pm \frac{\sqrt{3}}{2} \vec{H}\right|
\end{aligned}
$$


$=\sqrt{\frac{1}{4} \bar{H}^{2}+\frac{3}{4} \bar{H}^{\prime 2} \pm \frac{\sqrt{3}}{2} \bar{H} \bar{H}^{\prime} \cos \theta}$,

$\cos \theta=\left(\vec{H} \cdot \vec{H}^{\prime}\right) / \bar{H} \bar{H}^{\prime}$.

This shows that the classical background indeed has three degrees of freedom.

With the magnetic background (with $\bar{E}=0$ ) we have the effective potential given by

$V_{e f f}=\sum_{p}\left(\frac{\bar{H}_{p}^{2}}{3}+\frac{11 g^{2}}{48 \pi^{2}} \bar{H}_{p}^{2}\left(\ln \frac{g \bar{H}_{p}}{\mu^{2}}-c\right)\right)$.

We can renormalize the potential by defining a running coupling $\bar{g}^{2}\left(\bar{\mu}^{2}\right)$

$$
\begin{gathered}
\left.\forall_{p} \frac{\partial^{2} V_{\text {eff }}}{\partial \bar{H}_{p}^{2}}\right|_{\bar{H}_{1}=\bar{H}_{2}=\bar{H}_{3}=\bar{\mu}^{2}}=\frac{g^{2}}{\bar{g}^{2}} \\
=1+\frac{11 g^{2}}{16 \pi^{2}}\left(\ln \frac{\bar{\mu}^{2}}{\mu^{2}}-c+\frac{5}{4}\right),
\end{gathered}
$$

from which we retrieve the SU(3) QCD $\beta$-function which assures the asymptotic freedom [53,54]

$\beta(\bar{\mu})=\bar{\mu} \frac{d \bar{g}}{d \bar{\mu}}=-\frac{11 \bar{g}^{3}}{16 \pi^{2}}$.

With this we have the renormalized potential

$V_{e f f}=\sum_{p}\left(\frac{\bar{H}_{p}^{2}}{3}+\frac{11 \bar{g}^{2}}{48 \pi^{2}} \bar{H}_{p}^{2}\left(\ln \frac{g \bar{H}_{p}}{\bar{\mu}^{2}}-c\right)\right)$.

We plot the effective potential for $\cos \theta=0$ in Fig. 8 and for $\cos \theta=1$ in Fig. 9 for comparison, where we have put $\bar{\mu}=1$ and $\bar{\alpha}_{s}=1$.

The potential has the absolute minimum at $\bar{H}_{1}=\bar{H}_{2}=$ $\bar{H}_{3}=H_{0}$ (or equivalently $\bar{H}=\bar{H}^{\prime}=H_{0}$ and $\cos \theta=0$ ),

$\left\langle\bar{H}_{1}\right\rangle=\left\langle\bar{H}_{2}\right\rangle=\left\langle\bar{H}_{3}\right\rangle=\frac{\bar{\mu}^{2}}{\bar{g}} \exp \left(-\frac{16 \pi^{2}}{11 \bar{g}^{2}}+\frac{3}{4}\right)=H_{0}$,

$V_{\text {min }}=-\frac{11 \bar{\mu}^{4}}{32 \pi^{2}} \exp \left(-\frac{32 \pi^{2}}{11 \bar{g}^{2}}+\frac{3}{2}\right)$.

This is the monopole condensation, or more precisely the monopole-antimonopole pair condensation, which generates the dimensional transmutation and the mass gap in $\mathrm{SU}(3)$ QCD.

Notice that when $\vec{H}$ and $\vec{H}^{\prime}$ are parallel (i.e., when $\cos \theta=$ 1) it has two degenerate minima at $\bar{H}=2^{1 / 3} H_{0}, \bar{H}^{\prime}=0$ and at $\bar{H}=2^{-2 / 3} H_{0}, \quad \bar{H}^{\prime}=\sqrt{3} \times 2^{-2 / 3} H_{0}$. This is shown in Fig. 9. In terms of $\left(\bar{H}_{1}, \bar{H}_{2}, \bar{H}_{3}\right)$ the two degenerate minima are given by $\left(2^{1 / 3} H_{0}, 4^{-1 / 3} H_{0}, 4^{-1 / 3} H_{0}\right)$ and $\left(4^{-1 / 3} H_{0}, 2^{1 / 3} H_{0}, 4^{-1 / 3} H_{0}\right)$.

It must be emphasized that the Weyl invariance plays a crucial role in the true $\mathrm{SU}$ (3) QCD vacuum. To see this, notice that the effective potential (63) is Weyl symmetric. So it is natural to assume that the minimum point has the maximal

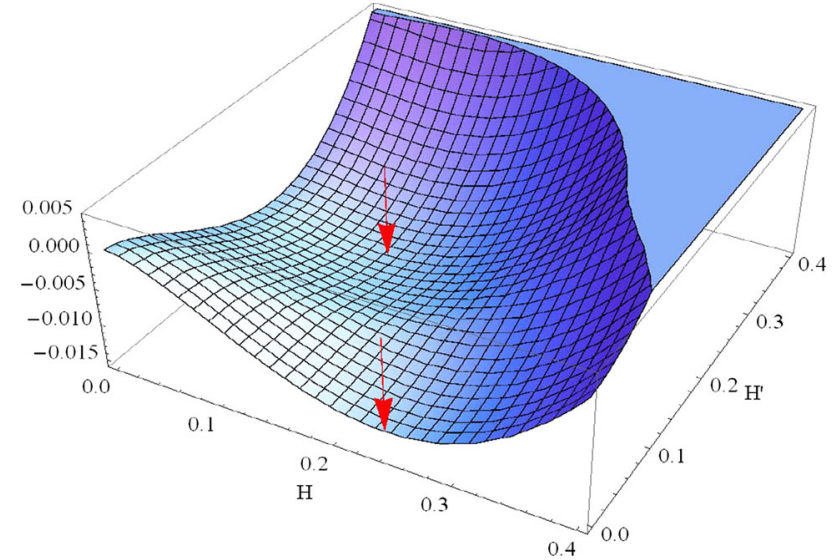

Fig. 9 The effective potential with $\cos \theta=1$, which has two degenerate minima

Weyl symmetry. This implies that the minimum point must form a singlet under the Weyl transformation, or $\bar{H}_{1}=\bar{H}_{2}=$ $\bar{H}_{3}$. This is fulfilled when $\vec{H}$ and $\vec{H}^{\prime}$ are orthogonal and $\vec{H}=$ $\bar{H}^{\prime}$. And this is exactly the true minimum shown in Fig. 8.

The above analysis demonstrates the followings. First, of course, SU(3) QCD has the stable monopole condensation which could be identified as the physical vacuum. Second, the monopole condensation naturally reproduces the asymptotic freedom. Third, the chromo-electric flux makes the pair annihilation of chromons. This confirms that essentially all qualitative features of the SU(2) QCD effective action translate to the $\mathrm{SU}(3) \mathrm{QCD}$ effective action, or in general SU(N) QCD effective action.

Obviously, just as in $\mathrm{SU}(2) \mathrm{QCD}$, the color reflection invariance plays the crucial role in SU(3) QCD. It is this symmetry which assures the the gauge invariance of the effective action and the stability of the monopole condensation. In retrospect this is exactly what we expected. Clearly the gauge invariance forbids colored objects from the physical spectrum in QCD, which is why we have the color confinement. But this gauge invariance, after the Abelian decomposition, becomes the color reflection invariance. So it is just natural that the color reflection invariance generates the monopole condensation which explains the color confinement.

On the other hand, there are new features in the SU(3) effective Lagrangian. First, the effective Lagrangian is characterized by three variables $\bar{H}_{p}$. This is understandable because $\mathrm{SU}(3)$ has three Casimir invariants. What is unexpected is that the monopole condensation given by $\vec{H}$ and $\vec{H}^{\prime}$ (or $\vec{E}$ and $\vec{E}^{\prime}$ ) in general can have different space orientation, and only when $\vec{H}$ and $\vec{H}^{\prime}$ are orthogonal and we have the true vacuum. As we pointed out, this is because the vacuum must have the maximal Weyl symmetry. 


\section{Discussion}

In this paper we have shown how to generalize the calculation of the SU(2) QCD effective action to that of the SU(3) QCD, with the help of the Weyl symmetric Abelian decomposition. Our result confirms that all essential features of the SU(2) QCD effective action remain unchanged in the SU(3) QCD. In particular we have the stable monopole condensation which generates the dimensional transmutation and the mass gap.

Our calculation should be contrasted with the old calculations which had critical defects [17-26]. The Savvidy vacuum has an intrinsic instability. Worse, it is not gauge invariant nor parity conserving. So it could not be the QCD vacuum. The Abelian decomposition allows us to remove these defects and obtain the stable monopole condensation.

In specific the Abelian decomposition gives us the following advantages. First, it decomposes QCD to the classical background and the quantum fluctuation gauge independently. In particular, it allows us to separate the gauge invariant and parity conserving monopole background gauge independently. Second, it reduces the non-Abelian gauge symmetry to the simple and discrete color reflection symmetry. This makes the implementation of the gauge invariance much easier. Third, it allows us to express the SU(3) QCD, or in general SU(N) QCD, in terms of the SU(2) QCD in a Weyl symmetric way. This effectively reduces the calculation of the SU(3) QCD or in general SU(N) QCD effective action to that of the SU(2) QCD effective action. These are the new features which were lacking in the old calculations.

But we emphasize that the monopole condensation should really be understood as the monopole-antimonopole condensation. This is because in QCD the monopole and antimonopole are gauge equivalent, since they are related by the gauge transformation [4-7,36].

This has a deep meaning. It has often been claimed that the color confinement in QCD comes from "the dual Meissner effect" generated by the monopole condensation [1-3]. We emphasize, however, that the confinement mechanism in QCD is not exactly dual to the Meissner effect which confines the magnetic flux in ordinary superconductor.

In superconductor the magnetic flux is screened by the supercurrent generated by the electron pairs, without the positron pairs. And obviously the Cooper pairs carry the electric charge. But in QCD the chromo-electric flux is confined by the monopole-antimonopole condensation, not by the supercurrent of monopole pairs. Besides, the monopoleantimonopole pairs have no magnetic charge. Moreover, the underlying mass generation mechanism in the Meissner effect is the spontaneous symmetry breaking (i.e., Higgs) mechanism. But in QCD it is the dynamical symmetry breaking mechanism without any ad hoc input mass scale. This tells that the two confining mechanisms are not exactly dual to each other.

An important consequence of this monopole-antimonopole condensation is that in QCD the colored flux which confines the $q \bar{q}$ pairs has no sense of helicity. As a result hadrons in the quark model have no parity doubling partners. This resolves the long standing problem of the parity doubling in hadron spectroscopy [60].

Moreover our result confirms that in the presence of chromo-electric background the effective action (56) has a negative imaginary part. This tells that the cheromo-electric flux has an instability which induces the pair annihilation, not the pair creation, of chromons [30-33,53-55]. This should be compared with the pair creation of electrons in the electric background in QED, which makes the screening of the electric charge [56-59]. The negative imaginary part in (56) tells that in QCD we have the anti-screening of color, which explains the asymptotic freedom.

Obviously the proof of the monopole condensation in QCD is very important from the theoretical point of view. But one may ask if there is any way to verify this monopole condensation experimentally. There might. To see this consider the Meissner effect in superconductor characterized by two scales, the correlation length of Cooper pair and the penetration length of magnetic field. Physically they describe the masses of the Higgs field and massive vector field. In other words, the spontaneous symmetry breaking generates two physical states which can be verified experimentally.

In QCD we could think of similar scales and similar phenomenon. For example, we could think of the correlation length of the monopole-antimonopole pair and the penetration length of the color flux, which could create two new states, the "magnetic" glueballs. In this logic we could have the $0^{++}$and $1^{++}$modes of the vacuum fluctuation of the monopole condensation, whose masses are fixed by two scales [4-7].

On the other hand, the situation is different in QCD. Here we have a dynamical symmetry breaking characterized by one scale, $\Lambda_{Q C D}$. Nevertheless, it is quite possible that the monopole condensation may have the vacuum fluctuation. This strongly suggests that the monopole condensation could generate one vacuum fluctuation mode which can be identified as the $0^{++}$monoball $[34,47,48]$. Moreover, there is a good candidate of the monoball, $f_{0}(500)$. With a broad decay width it has defied a simple classification, but could be an ideal candidate of the monoball.

The Abelian decomposition does many things. In the perturbative regime it decomposes the gluons to neurons and chromons, and decomposes the Feynman diagram. In nonperturbative QCD it proves the monopole dominance and demonstrates the monopole condensation. But it has another deep impact on hadron spectroscopy. It generalizes the quark 
model to the quark and chromon model which provides a new picture of hadrons, in particular the glueballs.

The identification of glueballs has been a big issue in QCD. The general wisdom is that QCD must have the glueballs made of gluons [61-66]. But the search for the glueballs has not been so successful for two reasons. First, theoretically there has been no consensus on how to construct the glueballs from the gluons. This made it difficult for us to predict what kind of glueballs we can expect.

The other reason is that experimentally it is not clear how to identify the glueballs. This is partly because the glueballs could mix with the quarkoniums, so that we must take care of the possible mixing to identify the glueballs experimentally $[47,48]$. This is why we have veryfew candidates of the glueballs so far, compared to huge hadron spectrum made of quarks [67].

The Abelian decomposition provides a totally new picture of glueball. Figure 4 tells that the chromons, just like the quarks, become the colored constituent of hadrons while the neurons play the role of the binding gluons. This means that we can identify the glueballs as the color reflection invariant bound states of chromons. This naturally generalizes the quark model to the quark and chromon model, and provides a simple picture of the quarkonium-glueball mixing.

This should be compared with other models of glueballs, in particular the bag model and the constituent gluon model [63-66]. Our quark and chromon model is fundamentally different from these in one important respect. They treat all gluons on the same footing, with no distinction between the binding gluons and the constituent gluons. But our model treats only the chromons as the constituent gluons, so that only the chromons become the constituent of the glueballs [4-7]. Moreover, this picture describes the glueball-quarkonium mixing successfully. The numerical analysis of the mixing in this picture below $2 \mathrm{GeV}$ shows that $f_{0}(1500), f_{2}(1950)$, $\eta(1405)$, and $\eta(1475)$ in $0^{++}, 2^{++}$, and $0^{-+}$sectors can be identified as predominantly the glueball states $[47,48]$.

In this paper we have neglected the quarks. We simply remark that the quark loop, just like the electron loop in QED, tend to diminish the asymptotic freedom. This is because the quark loop, unlike the chromon loop, generates the quark pair creation which makes the color screening. The reason is simple. The quark loop obeys the Fermi statistics but the chromon loop obeys the Bose statistics, so that the imaginary part they create in the chromo-electric background should have opposite signature.

But if the number of quarks are small enough, the asymptotic freedom holds. In fact we can show that exactly the same constraint on the number of quarks is needed to keep the asymptotic freedom.

In this paper we have calculated the one-loop effective action of QCD. Of course one could try to calculate the twoloop effective action of QCD using the Abelian decomposi- tion, which could also simplify the calculation greatly. On the other hand, the two-loop correction is not expected to change the one-loop result in any qualitative way.

Finally we emphasize that the underlying premise of this paper, the Abelian decomposition which shows the existence of two types of gluons which play totally different roles, is not just a mathematical proposition. It can actually be tested experimentally. This is because the neurons and chromons have different color factors, so that they have different jet structures. This means that QCD should actually have three different jets which can be distinguished by experiment, the quark jet, the neuron jet, and the chromon jet.

The prediction of the existence of the gluons and subsequent experimental confirmation the gluon jet were a great sucess for QCD [68-72]. Now the Abelian decomposition tells that there should be two types of gluon jets, the neuron jet and chromon jet. The experimental confirmation of the two types of gluon jets would be at least as important as the discovery of the gluon jet. The details of the two types of gluon jets will be discussed in a separate publication [73].

Acknowledgements The work is supported in part by National Research Foundation of Korea funded by the Ministry of Education (Grants 2015-R1D1A1A0-1057578 and 2018-R1D1A1B0-7045163), and by the Center for Quantum Spacetime at Sogang University.

Data Availability Statement This manuscript has associated data in a data repository. Data sharing not applicable to this article as no datasets were generated or analysed during the current study.

Open Access This article is distributed under the terms of the Creative Commons Attribution 4.0 International License (http://creativecomm ons.org/licenses/by/4.0/), which permits unrestricted use, distribution, and reproduction in any medium, provided you give appropriate credit to the original author(s) and the source, provide a link to the Creative Commons license, and indicate if changes were made. Funded by SCOAP $^{3}$.

\section{References}

1. Y. Nambu, Phys. Rev. D 10, 4262 (1974)

2. S. Mandelstam, Phys. Rep. 23C, 245 (1976)

3. A. Polyakov, Nucl. Phys. B 120, 429 (1977)

4. Y.M. Cho, Phys. Rev. D 21, 1080 (1980)

5. Y.S. Duan, M.L. Ge, Sci. Sin. 11, 1072 (1979)

6. Y.M. Cho, Phys. Rev. Lett. 46, 302 (1981)

7. Y.M. Cho, Phys. Rev. D 23, 2415 (1981)

8. G. 't Hooft, Nucl. Phys. B 190, 455 (1981)

9. Y.M. Cho, Phys. Rev. D 62, 074009 (2000)

10. S. Kato, K. Kondo, T. Murakami, A. Shibata, T. Shinohara, S. Ito, Phys. Lett. B 632, 326 (2006)

11. S. Kato, K. Kondo, T. Murakami, A. Shibata, T. Shinohara, S. Ito, Phys. Lett. B 645, 67 (2007)

12. S. Kato, K. Kondo, T. Murakami, A. Shibata, T. Shinohara, S. Ito, Phys. Lett. B 653, 101 (2007)

13. S. Kato, K. Kondo, T. Murakami, A. Shibata, T. Shinohara, S. Ito, Phys. Lett. B 669, 107 (2008)

14. N. Cundy, Y.M. Cho, W. Lee, J. Leem, Phys. Lett. B 729, 192 (2014) 
15. N. Cundy, Y.M. Cho, W. Lee, J. Leem, Nucl. Phys. B 895, 64 (2015)

16. S. Coleman, E. Weinberg, Phys. Rev. D 7, 1888 (1973)

17. G.K. Savvidy, Phys. Lett. B 71, 133 (1977)

18. N. Nielsen, P. Olesen, Nucl. Phys. B 144, 485 (1978)

19. N. Nielsen, P. Olesen, Nucl. Phys. B 160, 380 (1979)

20. C. Rajiadakos, Phys. Lett. B 100, 471 (1981)

21. W. Dittrich, M. Reuter, Phys. Lett. B 128, 321 (1983)

22. W. Dittrich, M. Reuter, Phys. Lett. B 144, 99 (1984)

23. M. Reuter, M.G. Schmidt, C. Schubert, Ann. Phys. 259, 313 (1997)

24. A. Yildiz, P. Cox, Phys. Rev. D 21, 1095 (1980)

25. M. Claudson, A. Yilditz, P. Cox, Phys. Rev. D 22, 2022 (1980)

26. J. Ambjorn, R. Hughes, Phys. Lett. B 113, 305 (1982)

27. F. Gliozzi, J. Scherk, D. Olive, Nucl. Phys. B 122, 253 (1977)

28. M. Green, J. Schwarz, E. Witten, Superstring Theory, vol. I (Cambridge University Press, Cambridge, 1987)

29. M. Kaku, Introduction to Superstrings (Springer, Berlin, 1988)

30. Y.M. Cho, D.G. Pak, Phys. Rev. D 65, 074027 (2002)

31. Y.M. Cho, H.W. Lee, D.G. Pak, Phys. Lett. B 525, 347 (2002)

32. Y.M. Cho, M.L. Walker, D.G. Pak, JHEP 05, 073 (2004)

33. Y.M. Cho, M.L. Walker, Mod. Phys. Lett A19, 2707 (2004)

34. Y.M. Cho, Franklin H. Cho, J.H. Yoon, Phys. Rev. D 87, 085025 (2013)

35. Y.M. Cho, Phys. Rev. Lett. 44, 1115 (1980)

36. Y.M. Cho, Phys. Lett. B 115, 125 (1982)

37. L. Faddeev, A. Niemi, Phys. Rev. Lett. 82, 1624 (1999)

38. L. Faddeev, A. Niemi, Phys. Lett. B 449, 214 (1999)

39. W.S. Bae, Y.M. Cho, S.W. Kim, Phys. Rev. D 65, 025005 (2001)

40. Y.M. Cho, Phys. Lett. B 644, 208 (2007)

41. S. Shabanov, Phys. Lett. B 458, 322 (1999)

42. S. Shabanov, Phys. Lett. B 463, 263 (1999)

43. H. Gies, Phys. Rev. D 63, 125023 (2001)

44. R. Zucchini, Int. J. Geom. Methods Mod. Phys. 1, 813 (2004)

45. K. Kondo, S. Kato, A. Shibata, T. Shinohara, Phys. Rep. 579, 1 (2015)

46. Y.M. Cho, Int. J. Mod. Phys. A 29, 1450013 (2014)

47. Y.M. Cho, X.Y. Pham, Pengming Zhang, Ju-Jun Xie, Li-Ping Zou, Phys. Rev. D 91, 114020 (2015)
48. Pengming Zhang, Li-Ping Zou, Y.M. Cho, Phys. Rev. D98, 096015 (2018)

49. B. de Witt, Phys. Rev. 162, 1195 (1967); 1239 (1967)

50. C. Itzikson, J. Zuber, Quantum Field Theory (McGraw-Hill, New York, 1985)

51. M. Peskin, D. Schroeder, An Introduction to Quantum Field Theory (Addison-Wesley, Boston, 1996)

52. Tsai, W., Yildiz, A.: Phys. Rev. D4, 3643 (1971); T Goldman and W. Tsai, ibid, 3648 (1971)

53. D. Gross, F. Wilczek, Phys. Rev. Lett. 30, 1343 (1973)

54. H. Politzer, Phys. Rev. Lett. 30, 1346 (1973)

55. V. Schanbacher, Phys. Rev. D 26, 489 (1982)

56. Y.M. Cho, D.G. Pak, Phys. Rev. Lett. 86, 1947 (2001)

57. Y.M. Cho, D.G. Pak, Phys. Rev. Lett. 91, 039151 (2001)

58. W.S. Bae, Y.M. Cho, D.G. Pak, Phys. Rev. D 64, 017303 (2001)

59. J. Schwinger, Phys. Rev. 82, 664 (1951)

60. Y.M. Cho, Nuovo Cimento 23A, 550 (1974)

61. H. Fritzsch, P. Minkowski, Nuovo Cimento 30A, 393 (1975)

62. P.G.O. Freund, Y. Nambu, Phys. Rev. Lett. 34, 1645 (1975)

63. R.L. Jaffe, K. Johnson, Phys. Lett. B 60, 201 (1976)

64. P. Roy, T. Walsh, Phys. Lett. 78B, 62 (1978)

65. J. Coyne, P. Fishbane, S. Meshkov, Phys. Lett. B 91, 259 (1980)

66. M. Chanowitz, Phys. Rev. Lett. 46, 981 (1981)

67. K. Olive et al. [Particle Data Group], Review of particle physics. Chin. Phys. C 38, 090001 (2014)

68. J. Ellis, M.K. Gaillard, G.G. Ross, Nucl. Phys. B 111, 253 (1976)

69. R. Brandelik et al. (TASSO Collaboration). Phys. Lett. B 86, 243 (1979)

70. D.P. Barber et al. (MARK-J Collaboration). Phys. Rev. Lett. 43, 830 (1979)

71. Ch. Berger et al. (PLUTO Collaboration), Phys. Lett. B 86, 418 (1979)

72. W. Bartel et al., JADE Collaboration, Phys. Lett. B 91, 142 (1980)

73. Y.M. Cho, F.H. Cho, P. Zhang. arXiv:1812.11300 [hep-ph] (to be published) 